4-1-2019

\title{
Efficient Class of Estimators for Finite Population Mean using Auxiliary Information in Two- Occasion Successive Sampling
}

G. N. Singh

Indian Institute of Technology (ISM)

Mohd Khalid

Indian Institute of Technology (ISM), mohdkhalidkhan4870@gmail.com

Follow this and additional works at: https://digitalcommons.wayne.edu/jmasm

Part of the Applied Statistics Commons, Social and Behavioral Sciences Commons, and the Statistical Theory Commons

\section{Recommended Citation}

Singh, G. N., \& Khalid, M. (2018). Efficient class of estimators for finite population mean using auxiliary information in two-occasion successive sampling. Journal of Modern Applied Statistical Methods, 17(2), eP2607. doi: 10.22237/jmasm/1554138114

This Regular Article is brought to you for free and open access by the Open Access Journals at DigitalCommons@WayneState. It has been accepted for inclusion in Journal of Modern Applied Statistical Methods by an authorized editor of DigitalCommons@WayneState. 


\section{Efficient Class of Estimators for Finite Population Mean using Auxiliary Information in Two-Occasion Successive Sampling}

\section{Cover Page Footnote}

Authors are thankful to the Indian Institute of Technology (Indian School of Mines), Dhanbad, for providing financial and necessary infrastructural support to carry out the present research work. 


\section{Efficient Class of Estimators for Finite Population Mean using Auxiliary Information in Two-Occasion Successive Sampling}

\author{
G. N. Singh \\ Indian Institute of Technology (ISM) \\ Dhanbad, India
}

\author{
Mohd Khalid \\ Indian Institute of Technology (ISM) \\ Dhanbad, India
}

In the case of sampling on two occasions, a class of estimators is considered which uses information on the first occasion as well as the second occasion in order to estimate the population means on the current (second) occasion. The usefulness of auxiliary information in enhancing the efficiency of this estimation is examined through the class of proposed estimators. Some properties of the class of estimators and a strategy of optimum replacement are discussed. The proposed class of estimators were empirically compared with the sample mean estimator in the case of no matching. The established optimum estimator, which is a linear combination of the means of the matched and unmatched portions of the sample at the current occasion, was empirically compared with the proposed class of estimators. Mutual comparisons of the proposed estimator were carried out. Suitable recommendations are made to the survey statistician for practical applications.

Keywords: Successive sampling, auxiliary information, bias, mean square error, optimum replacement strategy

\section{Introduction}

The theory of successive sampling has a long and rich history in sample surveys. When the value of the characteristics of interest of a finite population are subject to change over time, a survey carried out on a single occasion gives an idea only about the given occasion and can't give an idea about the (a) nature or rate of change of the characteristic at different occasions and (b) the average value of the characteristic over all occasions or most the recent occasions. Successive sampling, however, provides a tool for determining robust estimates. Successive (rotation)

doi: 10.22237/jmasm/1554138114 | Accepted: July 31, 2017; Published: April 1, 2019.

Correspondence: Mohd Khalid, mohdkhalidkhan4870@gmail.com 


\section{SINGH \& KHALID}

sampling was first coined by Jessen (1942) in the analysis of a survey on farm data. This theory was expanded by Patterson (1950), Eckler (1955), Cochran (1977), Gupta (1979), Das (1982), and Chaturvedi and Tripathi (1983).

Sen (1971) used this concept in designing the estimator of the current population mean on the current occasion by using information on two auxiliary variables which were easily available on the previous occasion. Sen $(1972,1973)$ extended this work for more than two auxiliary variables on the current occasion. V. K. Singh, Singh, and Shukla (1991) and G. N. Singh and Singh (2001) used the auxiliary information on the current occasion and proposed an estimator for the current population in two-occasion successive sampling. G. N. Singh (2003) extended this methodology for h-occasions successive sampling in the estimation of the current population mean in two-occasion successive sampling.

Sometimes, information on an auxiliary variable may be readily available on the first as well as the second occasion; for example, tonnage (or seat capacity) of each vehicle or ship is known in transportation surveys. More examples are available in survey literature, and may be used where the information on auxiliary variables are available on both occasions in two occasions successive sampling. Feng and Zou (1997), Biradar and Singh (2001), G. N. Singh (2005), G. N. Singh and Priyanka (2008), G. N. Singh and Karna (2009), H. P. Singh and Vishwakarma (2009), G. N. Singh and Prasad (2010), G. N. Singh, Prasad, and Karna (2011), G. N. Singh, Homa, and Murya (2013), H. P. Singh et al. (2015), and H. P. Singh, Kim, and Tarray (2016), among others, used auxiliary information on both occasions for estimating the population mean on the current (second) occasion in two-occasion successive sampling.

In some situations, information on several auxiliary variables may be readily available or may be made easily available by diverting some amounts of funds available in surveys. For example, to study the case of public health and welfare of a state or country, several factors are known that can be treated as an auxiliary variable, such as the number of beds, the number of doctors and supporting staff in different hospitals, amount of funds available for medicine, etc., may be known. Similarly, there are several pieces of information available, which if utilized appropriately can be led to improve the precision of the suggested estimators. The

purpose of this study is to propose some relevant class of estimators for estimating the current population mean in two-occasion sampling by utilizing the dynamic auxiliary variables available on both the occasions. 


\section{Formulation of the Proposed Estimators with Notation and Sample Structure}

Let $\mathrm{U}=\left(U_{1}, U_{2}, \ldots, U_{N}\right)$ be a finite population of size $N$ which has been sampled over two occasions and the character under study be denoted by $x(y)$ on the first (second) occasion. Assume the information on a varying auxiliary variable $z_{1}\left(z_{2}\right)$, whose population mean is known and is closely related to $x$ and $y$, is available on the first (second) occasion. A simple random sample (without replacement) of $n$ units is drawn on the first (second) occasion. When selecting the sample on the second (current) occasion, retain a (matched) random sub-sample of size $m=n \lambda$ from the sample selected on the first occasion and the remaining $u=n \mu$ units are replaced by a fresh selection under simple random sampling without replacement from the entire population so the sample size on the second (current) occasion is also $n$. $\lambda$ and $\mu(\lambda+\mu=1)$ are the fractions of the matched and fresh samples, respectively, on the second (current) occasion. Onwards, note the following notations:

$\bar{X}, \bar{Y}, \bar{Z}$ : The population means of the variables $x, y$, and $z$, respectively.

$\bar{Z}_{1}, \bar{Z}_{2}$ : The population means of the variables $z_{1}$ and $z_{2}$, respectively.

$\bar{x}_{n}, \bar{x}_{m}, \bar{y}_{u}, \bar{y}_{m}, \bar{z}_{u}, \bar{z}_{m}, \bar{z}_{n}, \bar{z}_{1 n}, \bar{z}_{2 u}, \bar{z}_{2 m}$ : The sample means of the respective variables based on the sample size shown in subscripts.

$C_{x}, C_{y}, C_{z}, C_{z_{1}}, C_{z_{2}}$ : The coefficient of variation of the variables given in the subscripts.

$\beta_{y x}, \beta_{y z}, \beta_{x z}, \beta_{x z_{1}}, \beta_{x z_{2}}, \beta_{y z_{1}}, \beta_{y z_{2}}$ : The population regression coefficient of the variables shown in the subscripts.

$\rho_{y x}, \rho_{y z}, \rho_{x z}, \rho_{y z_{1}}, \rho_{y z_{2}}, \rho_{x z_{1}}, \rho_{x z_{2}}, \rho_{z_{1} z_{2}}$ : The correlation coefficients between the variables shown in the subscripts.

$S_{x}^{2}, S_{y}^{2}, S_{z}^{2}, S_{z_{1}}^{2}, S_{z_{2}}^{2}$ : The population variances of the variables $x, y, z, z_{1}$, and $z_{2}$, respectively

\section{Proposed Estimators}

To estimate the population mean $\bar{Y}$ on the current (second) occasion, two sets of estimators are proposed. The first estimator is based on the sample size $u(=n \mu)$ drawn afresh on the second occasion and second estimator is based on the matched sample of size $m(=n \lambda)$ common to both occasions. The two sets of proposed estimators are defined below. 


\section{SINGH \& KHALID}

The estimator based on the fresh sample is defined as

$$
T_{i u}=\bar{y}_{u}+k_{i}\left(\bar{Z}_{2}-\bar{z}_{2 u}\right) ; \quad i=1,2
$$

and, by setting $i=1$ and $k_{1}=b_{y z_{2}}$, equation (1) becomes

$$
T_{1 u}=\bar{y}_{u}+b_{y z_{2}}\left(\bar{Z}_{2}-\bar{z}_{2 u}\right)
$$

By setting $i=2$ and $k_{2}=\bar{y}_{u} / \bar{z}_{2 u}$, equation (1) becomes

$$
T_{2 u}=\frac{\bar{y}_{u}}{\bar{z}_{2 u}} \bar{Z}_{2}
$$

The estimators based on the matched sample are defined as

$$
T_{j m}=\bar{y}_{m}+b_{y x}\left(x_{n}^{*}-\bar{x}_{n}\right)+b_{y z_{2}}\left(\bar{Z}_{2}-\bar{z}_{2 m}\right) \text {, }
$$

where $\bar{x}_{n}^{*}=\bar{x}_{n}+k_{j}^{*}\left(\bar{Z}_{1}-\bar{z}_{1 n}\right), j=1,2,3,4$.

Setting $j=1$ and $k_{1}^{*}=0$, equation (4) becomes

$$
T_{1 m}=\bar{y}_{m}+b_{y x}\left(\bar{x}_{n}-\bar{x}_{m}\right)+b_{y z_{2}}\left(\bar{Z}_{2}-\bar{z}_{2 m}\right) \text {. }
$$

Setting $j=2$ and $k_{2}^{*}=b_{x z_{1}}$, equation (4) becomes

$$
T_{2 m}=\bar{y}_{m}+b_{y x}^{m}\left(\left(\bar{x}_{n}+b_{x z_{1}}^{n}\left(\bar{Z}_{1}-\bar{z}_{1 n}\right)\right)-\bar{x}_{m}\right)+b_{y z_{2}}^{m}\left(\bar{Z}_{2}-\bar{z}_{2 m}\right) .
$$

Setting $j=3$ and $k_{3}^{*}=\bar{x}_{n} / \bar{z}_{1 n}$, equation (4) becomes

$$
T_{3 m}=\bar{y}_{m}+b_{y x}^{m}\left(\frac{\bar{x}_{n}}{\bar{z}_{1 n}} \bar{Z}_{1}-\bar{x}_{m}\right)+b_{y z_{2}}^{m}\left(\bar{Z}_{2}-\bar{z}_{2 m}\right),
$$

and by setting $j=4$ and $k_{3}^{*}=\bar{x}_{n} / \bar{Z}_{1}$, equation (4) becomes 


$$
T_{4 m}=\bar{y}_{m}+b_{y x}^{m}\left(\frac{\bar{x}_{n}}{\bar{Z}_{1}} \bar{z}_{1 n}-\bar{x}_{m}\right)+b_{y z_{2}}^{m}\left(\bar{Z}_{2}-\bar{z}_{2 m}\right)
$$

Considering the combination of the two sets of estimators $T_{i u}(\mathrm{i}=1,2)$ and $T_{j m}(j=1,2,3,4)$, the final estimators of the population mean on the current occasion are

$$
T_{i j}=\phi_{i j} T_{i u}+\left(1-\phi_{i j}\right) T_{j m}, \quad(i=1,2 \quad j=1,2,3,4),
$$

where the $\phi_{i j}$ are unknown constants to be determined so as to minimize the mean square error of the estimators $T_{i j}$ under certain criterions.

Remark 1. For estimating the population mean on each occasion, of the estimators, two sets $T_{i u}(i=1,2)$ are suitable, which implies that more belief on $T_{i u}$ could be shown by choosing $\phi_{i j}(i=1,2)$ as 1 (or close to 1 ). For estimating the change from one occasion to the next, the estimator $T_{j m}(j=1,2,3,4)$ could be more useful so $\phi_{i j}(j=1,2,3,4)$ might be chosen as 0 (or close to 0$)$. For asserting both the problems simultaneously, the suitable (optimum) choices of $\phi_{i j}(i=1,2$; $j=1,2,3,4)$ are desired.

\section{Properties of the Proposed Estimators $T_{i j}$}

The estimators $T_{i u}$ and $T_{j m}$ are regression, ratio, regression to regression, regressioncum ratio, and regression-cum product type in their respective structures, and they are biased estimators of the population mean $\bar{Y}$. Therefore, the final estimator $T_{i j}$ presented in equation (9) is also a biased estimator of the population mean $\bar{Y}$. The bias $\mathrm{B}\left(\right.$.) and mean square error $\mathrm{M}\left(\right.$.) of the proposed estimators $T_{i j}$ are derived up to the first order of sample size under the large sample assumption and using the following transformations:

$$
\begin{aligned}
& \bar{y}_{u}=\bar{Y}\left(1+e_{1}\right), \quad \bar{y}_{m}=\bar{Y}\left(1+e_{2}\right), \quad \bar{x}_{m}=\bar{X}\left(1+e_{3}\right), \quad \bar{x}_{n}=\bar{X}\left(1+e_{4}\right), \\
& \bar{z}_{2 u}=\bar{Z}_{2}\left(1+e_{5}\right), \quad \bar{z}_{2 m}=\bar{Z}_{2}\left(1+e_{6}\right), \quad \bar{z}_{1 n}=\bar{Z}_{1}\left(1+e_{7}\right), \quad s_{y z_{2}}(u)=S_{y z_{2}}\left(1+e_{8}\right), \\
& s_{z_{2}}^{2}(u)=S_{z_{2}}^{2}\left(1+e_{9}\right), \quad S_{y x}(m)=S_{y x}\left(1+e_{10}\right), \quad s_{x}^{2}(m)=S_{x}^{2}\left(1+e_{11}\right), \\
& s_{y z_{2}}(m)=S_{y z_{2}}\left(1+e_{12}\right), \quad s_{z_{2}}^{2}(m)=S_{z_{2}}^{2}\left(1+e_{13}\right), \quad s_{x z_{1}}(n)=S_{y z_{2}}\left(1+e_{14}\right), \\
& s_{z_{1}}^{2}(n)=S_{z_{2}}^{2}\left(1+e_{15}\right)
\end{aligned}
$$




\section{SINGH \& KHALID}

such that $\mathrm{E}\left(e_{k}\right)=0$ and $\left|e_{k}\right| \leq 1 \forall k=1,2, \ldots, 15$.

Under the above transformations the proposed estimators take the following form:

$$
\begin{gathered}
T_{1 u}=\bar{Y}\left(1+e_{1}\right)+\beta_{y z_{2}} \bar{Z}_{2}\left(-e_{5}+e_{5} e_{9}-e_{5} e_{8}\right) \\
T_{2 u}=\bar{Y}\left(1+e_{1}\right)\left(1+e_{5}\right)^{-1} \\
T_{1 m}=\bar{Y}\left(1+e_{2}\right)+\beta_{y x} \bar{X}\left(e_{4}-e_{3}-e_{4} e_{11}+e_{3} e_{11}+e_{4} e_{10}-e_{3} e_{10}\right) \\
-\beta_{y z_{2}} \bar{Z}_{2}\left(e_{6}-e_{6} e_{9}+e_{6} e_{8}\right) \\
T_{2 m}=\bar{Y}\left(1+e_{2}\right)+\beta_{y x} \bar{X}\left(e_{4}-e_{3}-e_{4} e_{11}+e_{3} e_{11}+e_{4} e_{10}-e_{3} e_{10}\right) \\
-\beta_{y x} \beta_{x z_{1}} \overline{Z_{1}}\left(e_{7}-e_{7} e_{11}+e_{7} e_{10}\right)-\beta_{y z_{2}} \bar{Z}_{2}\left(e_{6}-e_{6} e_{9}+e_{6} e_{8}\right) \\
T_{3 m}=\bar{Y}\left(1+e_{2}\right)+\beta_{y x} \bar{X}\left(e_{4}-e_{3}-e_{4} e_{11}+e_{3} e_{11}+e_{4} e_{10}-e_{3} e_{10}\right. \\
\left.-e_{7}-e_{7} e_{11}+e_{7} e_{10}-e_{4} e_{7}+e_{7}^{2}\right)-\beta_{y z_{2}} \bar{Z}_{2}\left(e_{6}-e_{6} e_{9}+e_{6} e_{8}\right) \\
T_{4 m}=\bar{Y}\left(1+e_{2}\right)+\beta_{y x} \bar{X}\left(e_{4}-e_{3}+e_{7}-e_{4} e_{11}+e_{3} e_{11}+e_{4} e_{7}-e_{7} e_{11}\right. \\
\left.+e_{4} e_{10}-e_{3} e_{10}+e_{7} e_{10}\right)-\beta_{y z_{2}} \bar{Z}_{2}\left(e_{6}-e_{6} e_{9}+e_{6} e_{8}\right)
\end{gathered}
$$

Theorem 1. The bias of the estimators $T_{i j}(i=1,2 ; j=1,2,3,4)$ up to the first order approximation is obtained as

$$
\mathrm{B}\left(T_{i j}\right)=\phi_{i j} \mathrm{~B}\left(T_{i u}\right)+\left(1-\phi_{i j}\right) \mathrm{B}\left(T_{j m}\right), \quad i=1,2 ; j=1,2,3,4,
$$

where

$$
\begin{gathered}
\mathrm{B}\left(T_{1 u}\right)=\left(\frac{1}{u}-\frac{1}{N}\right)\left(\frac{\mu_{0003} \mu_{0101}}{\mu_{0002}^{2}}-\frac{\mu_{0102}}{\mu_{0002}}\right) \\
\mathrm{B}\left(T_{2 u}\right)=\left(\frac{1}{u}-\frac{1}{N}\right)\left(\frac{\bar{Y} \mu_{0002}}{\bar{Z}_{2}^{2}}-\frac{\mu_{0101}}{\bar{Z}_{2}}\right)
\end{gathered}
$$




$$
\begin{aligned}
& \mathrm{B}\left(T_{1 m}\right)=\left(\frac{1}{n}-\frac{1}{m}\right)\left(\frac{\mu_{2100}}{\mu_{2000}}-\frac{\mu_{3000} \mu_{1100}}{\mu_{2000}^{2}}\right)+\left(\frac{1}{m}-\frac{1}{N}\right)\left(\frac{\mu_{0003} \mu_{0101}}{\mu_{0002}^{2}}-\frac{\mu_{0102}}{\mu_{0002}}\right) \\
& \mathrm{B}\left(T_{2 m}\right)=\left(\frac{1}{n}-\frac{1}{N}\right)\left(\frac{\mu_{0110} \mu_{1100}}{\mu_{2000} \mu_{0020}}-\frac{\mu_{1020} \mu_{1100} \mu_{0110}}{\mu_{0020}^{2} \mu_{2000}}-\frac{\mu_{1110} \mu_{1010}}{\mu_{2000} \mu_{0020}}\right) \\
&+\left(\frac{1}{n}-\frac{1}{m}\right)\left(\frac{\mu_{2100}}{\mu_{2000}}-\frac{\mu_{3000} \mu_{1100}}{\mu_{2000}^{2}}\right) \\
& \mathrm{B}\left(T_{3 m}\right)=\left(\frac{1}{n}-\frac{1}{N}\right)\left(\frac{\bar{X}}{\bar{Z}_{1}^{2}} \frac{\mu_{0020} \mu_{1100}}{\mu_{2000}}-\frac{\mu_{1010} \mu_{1100}}{\bar{Z}_{1} \mu_{2000}}+\frac{\mu_{1010} \mu_{1100}}{\bar{Z}_{1} \mu_{2000}^{2}}-\frac{\mu_{1110}}{\bar{Z}_{1} \mu_{2000}}\right) \\
&+\left(\frac{1}{n}-\frac{1}{m}\right)\left(\frac{\mu_{2100}}{\mu_{2000}}-\frac{\mu_{3000} \mu_{1100}}{\mu_{2000}^{2}}\right)+\left(\frac{1}{m}-\frac{1}{N}\right)\left(\frac{\mu_{0003} \mu_{0101}}{\mu_{0001}^{3}}-\frac{\mu_{0102}}{\mu_{0002}}\right) \\
& \mathrm{B}\left(T_{4 m}\right)=\left(\frac{1}{n}-\frac{1}{N}\right)\left(\frac{\mu_{1010} \mu_{1100}}{\bar{Z}_{1} \mu_{2000}}-\frac{\bar{X}}{\bar{Z}_{1}} \frac{\mu_{2010} \mu_{1100}}{\mu_{2000}}+\frac{\bar{X}}{\bar{Z}_{1}} \frac{\mu_{1110}}{\mu_{2000}}\right) \\
&+\left(\frac{1}{m}-\frac{1}{n}\right)\left(\frac{\mu_{3000} \mu_{1100}}{\mu_{2000}^{2}}-\frac{\mu_{2100}}{\mu_{2000}}\right)+\left(\frac{1}{m}-\frac{1}{N}\right)\left(\frac{\mu_{0003} \mu_{0101}}{\mu_{0001}^{3}}-\frac{\mu_{0102}}{\mu_{0002}}\right)
\end{aligned}
$$

where

$$
\mu_{p q r s}=\mathrm{E}\left[\left(x_{i}-\bar{X}\right)^{p}\left(y_{i}-\bar{Y}\right)^{q}\left(z_{1_{i}}-\bar{Z}\right)^{r}\left(z_{2_{i}}-\bar{Z}\right)^{s}\right],
$$

$p, q, r, s \geq 0$ are integers.

Proof: The bias of an estimator $T_{i j}$ is shown below:

$$
\begin{aligned}
\mathrm{B}\left(T_{i j}\right) & =\mathrm{E}\left[T_{i j}-\bar{Y}\right]=\phi_{i j} \mathrm{E}\left[T_{i u}-\bar{Y}\right]+\left(1-\phi_{i j}\right) \mathrm{E}\left[T_{j m}-\bar{Y}\right] \\
& =\phi_{i j} \mathrm{~B}\left(T_{i u}\right)+\left(1-\phi_{i j}\right) \mathrm{B}\left(T_{j m}\right)
\end{aligned}
$$

Using the transformations and retaining the terms up to the first order approximation, the expressions of $\mathrm{B}\left(T_{i u}\right)$ and $\mathrm{B}\left(T_{j m}\right)$ are obtained as in equations 


\section{SINGH \& KHALID}

(17)-(22). Hence, the expression of the bias of the estimator $T_{i j}$ are obtained as in equation (16).

Theorem 2. The mean square error of the estimators $T_{i j}(i=1,2 ; j=1,2,3,4)$ up to the first order approximation is derived as

$$
\mathrm{M}\left(T_{i j}\right)=\phi_{i j}^{2} \mathrm{M}\left(T_{i u}\right)+\left(1-\phi_{i j}\right)^{2} \mathrm{M}\left(T_{j m}\right)+2 \phi_{i j}\left(1-\phi_{i j}\right) \mathrm{C}\left(T_{i u}, T_{j m}\right),
$$

where

$$
\begin{gathered}
\mathrm{M}\left(T_{1 u}\right)=\left[\left(\frac{1}{u}-\frac{1}{N}\right)\left(1-\rho_{y z_{2}}^{2}\right)\right] S_{y}^{2} \\
\mathrm{M}\left(T_{2 u}\right)=\left[\left(\frac{1}{u}-\frac{1}{N}\right)\left(2-2 \rho_{y z_{2}}\right)\right] S_{y}^{2} \\
\mathrm{M}\left(T_{1 m}\right)=\left[\left(\frac{1}{m}-\frac{1}{N}\right)+\left(\frac{1}{n}-\frac{1}{m}\right) \rho_{y x}^{2}-\left(\frac{1}{m}-\frac{1}{N}\right) \rho_{y z_{2}}^{2}\right. \\
\mathrm{M}\left(T_{2 m}\right)=\left[\left(\frac{1}{m}-\frac{1}{N}\right)-\left(\frac{1}{m}-\frac{1}{n}\right) \rho_{y x}^{2}+\left(\frac{1}{n}-\frac{1}{N}\right) \rho_{y z_{1}}^{2} \rho_{y x}^{2}-\left(\frac{1}{m}-\frac{1}{N}\right) \rho_{x z_{2}}^{2} \rho_{y z_{2}} \rho_{y x}\right] S_{y}^{2} \\
-2\left(\frac{1}{n}-\frac{1}{N}\right) \rho_{x z_{1}} \rho_{x z_{1}} \rho_{y x}-2\left(\frac{1}{n}-\frac{1}{m}\right) \rho_{y z_{2}} \rho_{x z_{2}} \rho_{y x} \\
\left.+2\left(\frac{1}{n}-\frac{1}{N}\right) \rho_{y z_{2}} \rho_{x z_{1}} \rho_{y x} \rho_{z_{1} z_{2}}\right] S_{y}^{2}
\end{gathered}
$$




\section{EFFICIENT CLASS OF ESTIMATORS IN TWO-OCCASION SAMPLING}

$$
\begin{aligned}
& \mathrm{M}\left(T_{3 m}\right)=\left[\left(\frac{1}{m}-\frac{1}{N}\right)+\left(\frac{1}{m}-\frac{1}{N}\right) \rho_{y x}^{2}+2\left(\frac{1}{n}-\frac{1}{m}\right) \rho_{y x}^{2}-\left(\frac{1}{m}-\frac{1}{N}\right) \rho_{y z_{2}}^{2}\right. \\
& -2\left(\frac{1}{n}-\frac{1}{N}\right) \rho_{y z_{1}} \rho_{y x}-2\left(\frac{1}{n}-\frac{1}{m}\right) \rho_{y z_{2}} \rho_{x z_{2}} \rho_{y x} \\
& \left.+2\left(\frac{1}{n}-\frac{1}{N}\right) \rho_{y z_{2}} \rho_{y x} \rho_{z_{1} z_{2}}\right] S_{y}^{2} \\
& \mathrm{M}\left(T_{4 m}\right)=\left[\left(\frac{1}{m}-\frac{1}{N}\right)-\left(\frac{1}{m}-\frac{1}{N}\right) \rho_{y x}^{2}+2\left(\frac{1}{n}-\frac{1}{m}\right) \rho_{y x}^{2}-\left(\frac{1}{m}-\frac{1}{N}\right) \rho_{y z_{2}}^{2}\right. \\
& +2\left(\frac{1}{n}-\frac{1}{N}\right) \rho_{y z_{1}} \rho_{y x}-2\left(\frac{1}{n}-\frac{1}{m}\right) \rho_{y z_{2}} \rho_{x z_{2}} \rho_{y x} \\
& \left.-2\left(\frac{1}{n}-\frac{1}{N}\right) \rho_{y z_{2}} \rho_{y x} \rho_{z_{1} z_{2}}\right] S_{y}^{2} \\
& \mathrm{C}\left(T_{1 u}, T_{1 m}\right)=\mathrm{E}\left[\left(T_{1 u}-\bar{Y}\right)\left(T_{1 m}-\bar{Y}\right)\right]=\frac{S_{y}^{2}}{N}\left(1-\rho_{y z_{2}}^{2}\right) \\
& \mathrm{C}\left(T_{1 u}, T_{2 m}\right)=\mathrm{E}\left[\left(T_{1 u}-\bar{Y}\right)\left(T_{2 m}-\bar{Y}\right)\right] \\
& =\frac{S_{y}^{2}}{N}\left(1-\rho_{y x} \rho_{x z_{1}} \rho_{y z_{1}}-\rho_{y z_{2}}^{2}+\rho_{y x} \rho_{y z_{2}} \rho_{x z_{1}} \rho_{z_{1} z_{2}}\right) \\
& \mathrm{C}\left(T_{1 u}, T_{3 m}\right)=\mathrm{E}\left[\left(T_{1 u}-\bar{Y}\right)\left(T_{3 m}-\bar{Y}\right)\right]=\frac{S_{y}^{2}}{N}\left(1-\rho_{y x} \rho_{x z_{1}}-\rho_{y z_{2}}^{2}+\rho_{y x} \rho_{y z_{2}} \rho_{z_{1} z_{2}}\right) \\
& \mathrm{C}\left(T_{1 u}, T_{4 m}\right)=\mathrm{E}\left[\left(T_{1 u}-\bar{Y}\right)\left(T_{4 m}-\bar{Y}\right)\right]=\frac{S_{y}^{2}}{N}\left(1+\rho_{y x} \rho_{y z_{1}}-\rho_{y z_{2}}^{2}+\rho_{y x} \rho_{y z_{2}} \rho_{z_{1} z_{2}}\right) \\
& \mathrm{C}\left(T_{2 u}, T_{1 m}\right)=\mathrm{E}\left[\left(T_{2 u}-\bar{Y}\right)\left(T_{1 m}-\bar{Y}\right)\right]=\frac{S_{y}^{2}}{N}\left(1-\rho_{y z_{2}}^{2}\right) \\
& \mathrm{C}\left(T_{2 u}, T_{2 m}\right)=\mathrm{E}\left[\left(T_{2 u}-\bar{Y}\right)\left(T_{2 m}-\bar{Y}\right)\right] \\
& =\frac{S_{y}^{2}}{N}\left(1-\rho_{y x} \rho_{x z_{1}} \rho_{y z_{1}}-\rho_{y z_{2}}^{2}+\rho_{y x} \rho_{x z_{1}} \rho_{z_{1} z_{2}}\right)
\end{aligned}
$$




$$
\mathrm{C}\left(T_{2 u}, T_{3 m}\right)=\mathrm{E}\left[\left(T_{2 u}-\bar{Y}\right)\left(T_{3 m}-\bar{Y}\right)\right]=\frac{S_{y}^{2}}{N}\left(1-\rho_{y x} \rho_{y z_{1}}+\rho_{y x} \rho_{z_{1} z_{2}}-\rho_{y z_{2}}^{2}\right)
$$

and

$$
\mathrm{C}\left(T_{2 u}, T_{4 m}\right)=\mathrm{E}\left[\left(T_{2 u}-\bar{Y}\right)\left(T_{4 m}-\bar{Y}\right)\right]=\frac{S_{y}^{2}}{N}\left(1+\rho_{y x} \rho_{y z_{1}}-\rho_{y x} \rho_{z_{1} z_{2}}-\rho_{y z_{2}}^{2}\right) .
$$

Proof: $\quad$ The mean square error of the estimators $T_{i j}(i=1,2 ; j=1,2,3,4)$ are given by

$$
\begin{aligned}
\mathrm{M}\left(T_{i j}\right) & =\mathrm{E}\left[T_{i j}-\bar{Y}\right]=\mathrm{E}\left[\phi_{i j}\left(T_{i u}-\bar{Y}\right)+\left(1-\phi_{i j}\right)\left(T_{j m}-\bar{Y}\right)\right]^{2} \\
& =\phi_{i j}^{2} \mathrm{M}\left(T_{i u}\right)+\left(1-\phi_{i j}\right)^{2} \mathrm{M}\left(T_{j m}\right)+2 \phi_{i j}\left(1-\phi_{i j}\right) \mathrm{C}\left(T_{i u}, T_{j m}\right)
\end{aligned}
$$

where

$$
\begin{aligned}
\mathrm{M}\left(T_{i u}\right) & =\mathrm{E}\left[T_{i u}-\bar{Y}\right]^{2}, \quad \mathrm{M}\left(T_{j m}\right)=\mathrm{E}\left[T_{j m}-\bar{Y}\right]^{2}, \\
\mathrm{C}\left(T_{i u}, T_{j m}\right) & =\mathrm{E}\left[\left(T_{i u}-\bar{Y}\right)\left(T_{j m}-\bar{Y}\right)\right]
\end{aligned}
$$

Using the transformation and retaining the terms up to the first order approximation, the expressions of $\mathrm{M}\left(T_{i u}\right), \mathrm{M}\left(T_{j m}\right)$, and $\mathrm{C}\left(T_{i u}, T_{j m}\right)$ are obtained as in equation (24)-(37). Hence, the expression of the bias of the estimators $T_{i j}$ are obtained as in equation (23).

Remark 2. Because $x$ and $y$ denote the same study variable over two occasions and $z_{1}, z_{2}$ are auxiliary variables correlated to $x$ and $y$, looking on the stability nature viz. Reddy (1978), the coefficients of variation of $y, x, z_{1}$, and $z_{2}$ in the population are considered to be approximately equal, i.e. $C_{x}=C_{z_{1}}=C_{z_{2}}=C_{y}$.

\section{Minimum Mean Square Error of the Estimators $T_{i j}$}

Because the mean square error of the estimators $T_{i j}$ in equation (23) are a function of the unknown constants $\phi_{i j}(i=1,2 ; j=1,2,3,4)$, it is minimized with respect to $\phi_{i j}$ and subsequently the optimum value of $\phi_{i j}$, say $\left(\phi_{i j}\right)_{\mathrm{opt}}$, is obtained as 


\section{EFFICIENT CLASS OF ESTIMATORS IN TWO-OCCASION SAMPLING}

$$
\left(\phi_{i j}\right)_{\mathrm{opt}}=\frac{\mathrm{M}\left(T_{j m}\right)-\mathrm{C}\left(T_{i u}, T_{j m}\right)}{\mathrm{M}\left(T_{i u}\right)+\mathrm{M}\left(T_{j m}\right)-2 \mathrm{C}\left(T_{i u}, T_{j m}\right)}, \quad i=1,2 ; j=1,2,3,4 .
$$

Substituting the value of $\left(\phi_{i j}\right)_{\text {opt }}$ from equation (38) in equation (23) yields the optimum (minimum) mean square error of the estimators $T_{i j}$,

$$
\mathrm{M}\left(T_{i j}\right)_{\mathrm{opt}}=\frac{\mathrm{M}\left(T_{i u}\right) \mathrm{M}\left(T_{j m}\right)-\left[\mathrm{C}\left(T_{i u}, T_{j m}\right)\right]^{2}}{\mathrm{M}\left(T_{i u}\right)+\mathrm{M}\left(T_{j m}\right)-2 \mathrm{C}\left(T_{i u}, T_{j m}\right)}, \quad i=1,2 ; j=1,2,3,4 .
$$

Substituting the values of $\mathrm{M}\left(T_{i u}\right), \mathrm{M}\left(T_{j m}\right)$, and $\mathrm{C}\left(T_{i u}, T_{j m}\right)$ from equations (24)(37) in equations (38) and (39), we get the simplified values of $\left(\phi_{i j}\right)_{\mathrm{opt}}$ and $\mathrm{M}\left(T_{i j}\right)_{\mathrm{opt}}$ as

$$
\begin{gathered}
\left(\phi_{11}\right)_{\mathrm{opt}}=\frac{\mu_{11}\left[A_{4}-\mu_{11} A_{2}\right]}{A_{3}+\mu_{11} A_{5}+\mu_{11}^{2} A_{2}} \\
\mathrm{M}\left(T_{11}\right)_{\mathrm{opt}}=\frac{\left[A_{6}+\mu_{11} A_{7}+\mu_{11}^{2} A_{8}\right]}{\left[A_{3}+\mu_{11} A_{5}+\mu_{11}^{2} A_{2}\right]} \frac{S_{y}^{2}}{n} \\
\left(\phi_{12}\right)_{\mathrm{opt}}=\frac{\mu_{12}\left[A_{12}-\mu_{12} A_{13}\right]}{A_{3}+\mu_{12} A_{14}+\mu_{12}^{2} A_{15}} \\
\mathrm{M}\left(T_{12}\right)_{\mathrm{opt}}=\frac{\left[A_{16}+\mu_{12} A_{17}+\mu_{12}^{2} A_{18}\right]}{\left[A_{3}+\mu_{12} A_{14}+\mu_{12}^{2} A_{15}\right]} \frac{S_{y}^{2}}{n} \\
\left(\phi_{13}\right)_{\mathrm{opt}}=\frac{\mu_{13}\left[A_{22}-\mu_{13} A_{23}\right]}{A_{3}+\mu_{12} A_{24}+\mu_{13}^{2} A_{25}} \\
\mathrm{M}\left(T_{13}\right)_{\mathrm{opt}}=\frac{\left[A_{26}+\mu_{13} A_{27}+\mu_{13}^{2} A_{28}\right]}{\left[A_{3}+\mu_{13} A_{24}+\mu_{13}^{2} A_{25}\right]} \frac{S_{y}^{2}}{n}
\end{gathered}
$$




$$
\begin{aligned}
\left(\phi_{14}\right)_{\mathrm{opt}} & =\frac{\mu_{14}\left[A_{33}-\mu_{14} A_{34}\right]}{A_{3}+\mu_{14} A_{35}+\mu_{14}^{2} A_{36}} \\
\mathrm{M}\left(T_{14}\right)_{\mathrm{opt}}= & \frac{\left[A_{37}+\mu_{14} A_{38}+\mu_{14}^{2} A_{39}\right]}{\left[A_{3}+\mu_{14} A_{35}+\mu_{14}^{2} A_{36}\right]} \frac{S_{y}^{2}}{n} \\
\left(\phi_{21}\right)_{\mathrm{opt}} & =\frac{\mu_{21}\left[A_{4}-\mu_{21} A_{2}\right]}{A_{40}+\mu_{21} A_{41}+\mu_{21}^{2} A_{42}} \\
\mathrm{M}\left(T_{21}\right)_{\mathrm{opt}}= & \frac{\left[A_{43}+\mu_{21} A_{44}+\mu_{21}^{2} A_{45}\right]}{\left[A_{40}+\mu_{21} A_{41}+\mu_{21}^{2} A_{42}\right]} \frac{S_{y}^{2}}{n} \\
\mathrm{M}\left(T_{24}\right)_{\mathrm{opt}}= & \frac{\left[A_{67}+\mu_{24} A_{68}+\mu_{24}^{2} A_{69}\right]}{\left[A_{40}+\mu_{24} A_{65}+\mu_{24}^{2} A_{66}\right]} \frac{S_{y}^{2}}{n} \\
\left(\phi_{22}\right)_{\mathrm{opt}} & =\frac{\mu_{22}\left[A_{47}-\mu_{22} A_{48}\right]}{A_{40}+\mu_{22} A_{49}+\mu_{22}^{2} A_{50}} \\
\mathrm{M}\left(T_{23}\right)_{\mathrm{opt}}= & \frac{\left[A_{59}+\mu_{23} A_{60}+\mu_{23}^{2} A_{61}\right]}{\left[A_{40}+\mu_{23} A_{57}+\mu_{23}^{2} A_{58}\right]} \frac{S_{y}^{2}}{n} \\
\mathrm{M}\left(T_{22}\right)_{\mathrm{opt}}= & \frac{\mu_{24}\left[A_{63}-\mu_{24} A_{64}\right]}{\left.A_{51}+\mu_{22} A_{52}+\mu_{22}^{2} A_{53}\right]} \frac{S_{y}^{2}}{n} \\
& =\frac{\mu_{23}\left[A_{55}-\mu_{23} A_{56}\right]}{A_{40}+\mu_{23} A_{49}+\mu_{22}^{2} A_{50}+\mu_{23}^{2} A_{58}} \\
&
\end{aligned}
$$

where $\mu_{i j}=u / n$ and 


\section{EFFICIENT CLASS OF ESTIMATORS IN TWO-OCCASION SAMPLING}

$$
\begin{aligned}
& A_{1}=1-\rho_{y x}^{2}-\rho_{y z_{2}}^{2}+2 \rho_{y z_{2}}^{2} \rho_{y x} \rho_{x z_{2}}, A_{2}=\rho_{y x}^{2}-2 \rho_{y x} \rho_{y z_{2}} \rho_{x z_{2}}, A_{3}=1-\rho_{y z_{2}}^{2}, A_{4}=A_{1}+A_{2} \text {, } \\
& A_{5}=A_{4}-A_{3}, A_{6}=A_{3}\left(A_{4}-A_{3} f\right), A_{7}=-A_{1} A_{3} f-A_{2} A_{3}-A_{2} A_{3} f+A_{3}^{2} f, A_{8}=A_{2} A_{3} f \text {, } \\
& A_{9}=\rho_{y x}^{2}+\rho_{y x}^{2} \rho_{x z_{1}}^{2}-2 \rho_{y x} \rho_{y z_{1}} \rho_{x z_{1}}-2 \rho_{y x} \rho_{x z_{1}} \rho_{x z_{2}}+2 \rho_{y x} \rho_{y z_{2}} \rho_{x z_{1}} \rho_{z_{1} z_{2}} \text {, } \\
& A_{10}=1+\rho_{y x}^{2} \rho_{x z_{1}}^{2}-\rho_{y z_{2}}^{2}-2 \rho_{x z_{1}} \rho_{y x} \rho_{y z_{1}}+2 \rho_{y z_{2}} \rho_{y x} \rho_{x z_{1}} \rho_{z_{1} z_{2}} \text {, } \\
& A_{11}=1-\rho_{x z_{1}} \rho_{y x} \rho_{y z_{1}}-\rho_{y z_{2}}^{2}+2 \rho_{y z_{2}} \rho_{y x} \rho_{x z_{1}} \rho_{z_{1} z_{2}}, A_{12}=A_{1}+A_{9}-A_{10} f+A_{11} f \text {, } \\
& A_{13}=A_{9}-A_{10} f+A_{11} f, A_{14}=-A_{3} f+A_{1}+A_{9}-A_{10} f+2 A_{11} f \text {, } \\
& A_{15}=A_{3} f-A_{9}+A_{10} f-2 A_{11} f, A_{16}=A_{1} A_{3}+A_{9} A_{3}-A_{10} A_{3} f \text {, } \\
& A_{17}=-A_{3} A_{9}+A_{3} A_{10} f-A_{3} A_{1} f-A_{3} A_{9} f+A_{10} A_{3} f^{2}-A_{11}^{2} f^{2} \text {, } \\
& A_{18}=A_{3} A_{9} f-A_{10} A_{3} f^{2}+A_{11}^{2} f^{2}, A_{19}=2 \rho_{y x}^{2}-2 \rho_{y x} \rho_{y z_{1}}-2 \rho_{y x} \rho_{y z_{2}} \rho_{x z_{2}}+2 \rho_{y z_{2}} \rho_{y x} \rho_{z_{1} z_{2}} \text {, } \\
& A_{20}=1+\rho_{y x}^{2}-\rho_{y z_{2}}^{2}-2 \rho_{y x} \rho_{y z_{1}}+2 \rho_{y z_{2}} \rho_{y x} \rho_{x z_{2}}, A_{21}=1-\rho_{y x} \rho_{y z_{1}}-\rho_{y z_{2}}^{2}+\rho_{y z_{2}} \rho_{y x} \rho_{x z_{2}} \text {, } \\
& A_{22}=A_{1}+A_{19}-A_{20} f+A_{21} f, A_{23}=A_{19}-A_{20} f+A_{21} f \text {, } \\
& A_{24}=-A_{3}-A_{3} f+A_{1}+A_{19}-A_{20} f+2 A_{21} f, A_{25}=A_{3} f-A_{19}+A_{20} f-2 A_{21} f \text {, } \\
& A_{26}=A_{1} A_{3}+A_{3} A_{19}-A_{3} A_{20} f \text {, } \\
& A_{27}=-A_{3} A_{19}+A_{3} A_{20} f-A_{3} A_{1} f-A_{3} A_{19} f+A_{20} A_{3} f^{2}-A_{21}^{2} f^{2} \text {, } \\
& A_{28}=A_{3} A_{19} f-A_{20} A_{3} f^{2}+A_{11}^{2} f^{2}, A_{29}=1-3 \rho_{y x}^{2}-\rho_{y z_{2}}^{2}+2 \rho_{y z_{2}}^{2} \rho_{y x} \rho_{x z_{2}} \text {, } \\
& A_{30}=2 \rho_{y x}^{2}+2 \rho_{y x} \rho_{y z_{1}}-2 \rho_{y x} \rho_{y z_{2}} \rho_{x z_{2}}-2 \rho_{y z_{2}} \rho_{y x} \rho_{z_{1} z_{2}} \text {, } \\
& A_{31}=1-\rho_{y x}^{2}-\rho_{y z_{2}}^{2}+2 \rho_{y x} \rho_{y z_{1}}-2 \rho_{y z_{2}} \rho_{y x} \rho_{z_{1} z_{2}}, A_{32}=1+\rho_{y x} \rho_{y z_{1}}-\rho_{y z_{2}}^{2}+\rho_{y z_{2}} \rho_{y x} \rho_{z_{1} z_{2}} \text {, } \\
& A_{33}=A_{29}+A_{30}-A_{31} f+A_{32} f, A_{34}=A_{30}-A_{31} f+A_{32} f \text {, } \\
& A_{35}=-A_{3}-A_{3} f+A_{29}+A_{30}-A_{31} f+2 A_{32} f, A_{36}=A_{3} f-A_{30} A_{31} f-2 A_{32} f \text {, } \\
& A_{37}=A_{29} A_{3}+A_{30} A_{3}-A_{31} A_{3} f \text {, } \\
& A_{38}=-A_{3} A_{30}+A_{3} A_{31} f-A_{3} A_{29} f-A_{3} A_{30} f+A_{31} A_{3} f^{2}-A_{32}^{2} f^{2} \text {, } \\
& A_{39}=A_{3} A_{30} f-A_{31} A_{3} f^{2}+A_{32}^{2} f^{2}, A_{40}=2\left(1-\rho_{y z_{2}}\right) \text {, } \\
& A_{41}=-A_{40}-A_{40} f+A_{1}+A_{2}+A_{3} f, A_{42}=A_{40} f-A_{2}-A_{3} f \text {, } \\
& A_{43}=A_{1} A_{40}+A_{2} A_{40}-A_{3} A_{40} f \text {, } \\
& A_{44}=-A_{40} A_{2}+A_{40} A_{3} f-A_{40} A_{1} f-A_{40} A_{2} f+A_{3} A_{40} f^{2}-A_{3}^{2} f^{2} \text {, } \\
& A_{45}=A_{40} A_{2} f-A_{3} A_{40} f^{2}+A_{3}^{2} f^{2}, A_{46}=1-\rho_{y z_{2}}^{2}-\rho_{y x} \rho_{y z_{1}} \rho_{x z_{1}}+\rho_{y x} \rho_{z_{1} z_{2}} \text {, } \\
& A_{47}=A_{1}+A_{9}-A_{10} f+A_{46} f, A_{48}=A_{9}-A_{10} f+A_{46} f \text {, } \\
& A_{49}=-A_{40}-A_{40} f+A_{1}+A_{9}-A_{10} f+2 A_{46} f, A_{50}=A_{40} f-A_{9}+A_{10} f-2 A_{46} f \text {, }
\end{aligned}
$$




$$
\begin{aligned}
& A_{51}=A_{1} A_{40}+A_{9} A_{10}-A_{10} A_{40} f, \\
& A_{52}=-A_{40} A_{9}+A_{40} A_{10} f-A_{40} A_{1} f-A_{40} A_{9} f+A_{10} A_{40} f^{2}-A_{46}^{2} f^{2}, \\
& A_{53}=A_{40} A_{9} f-A_{10} A_{40} f^{2}+A_{46}^{2} f^{2}, A_{54}=1-\rho_{y z_{2}}^{2}-\rho_{y x} \rho_{y z_{1}}+\rho_{y x} \rho_{z_{1} z_{2}}, \\
& A_{55}=A_{1}+A_{19}-A_{20} f+A_{54} f, A_{56}=A_{19}-A_{20} f+A_{54} f, \\
& A_{57}=-A_{40}-A_{40} f+A_{1}+A_{19}-A_{20} f+2 A_{54} f, A_{58}=A_{40} f-A_{19}+A_{20} f-2 A_{54} f, \\
& A_{59}=A_{1} A_{40}+A_{19} A_{40}-A_{20} A_{40} f, \\
& A_{60}=-A_{40} A_{19}+A_{40} A_{20} f-A_{40} A_{1} f-A_{40} A_{19} f+A_{20} A_{40} f^{2}-A_{54}^{2} f^{2}, \\
& A_{61}=A_{40} A_{19} f-A_{20} A_{40} f^{2}+A_{54}^{2} f^{2}, A_{62}=1-\rho_{y z_{2}}^{2}+\rho_{y x} \rho_{y z_{1}}-\rho_{y x} \rho_{z_{1} z_{2}}, \\
& A_{63}=A_{29}+A_{30}-A_{31} f+A_{62} f, A_{64}=A_{30}-A_{31} f+A_{62} f, \\
& A_{65}=-A_{40}-A_{40} f+A_{29}+A_{30}-A_{31} f+2 A_{62} f, A_{66}=A_{40} f-A_{30}+A_{31} f-2 A_{62} f, \\
& A_{67}=A_{29} A_{40}+A_{30} A_{40}-A_{31} A_{40} f, \\
& A_{68}=-A_{40} A_{30}+A_{40} A_{31} f-A_{40} A_{29} f-A_{40} A_{30} f+A_{31} A_{40} f^{2}-A_{62}^{2} f^{2}, \\
& A_{69}=A_{40} A_{30} f-A_{31} A_{40} f^{2}+A_{62}^{2} f^{2},
\end{aligned}
$$

with $f=n / N$.

\section{Optimum Replacement Strategy of the Estimator $T_{i j}$}

To obtain the optimum values of $\mu_{i j}(i=1,2 ; j=1,2,3,4)$ (the fraction of the sample to be drawn afresh on the current occasion) so that the population mean $\bar{Y}$ may be estimated with maximum precision and minimum cost, minimize the mean square error of $T_{i j}$ given in equations (41), (43), (45), (47), (49), (51), (53), and (55) with respect to $\mu_{i j}$, which yields a quadratic equation in $\mu_{i j}$. The respective solutions of $\mu_{i j}$, say $\hat{\mu}_{i j}$, are given below:

$$
\begin{aligned}
& T_{1} \hat{\mu}_{11}^{2}+2 T_{2} \hat{\mu}_{11}+T_{3}=0 \\
& \hat{\mu}_{11}=\frac{-T_{2} \pm \sqrt{T_{2}^{2}-T_{1} T_{3}}}{T_{1}} \\
& P_{1} \hat{\mu}_{12}^{2}+2 P_{2} \hat{\mu}_{12}+P_{3}=0
\end{aligned}
$$




\section{EFFICIENT CLASS OF ESTIMATORS IN TWO-OCCASION SAMPLING}

$$
\begin{aligned}
& \hat{\mu}_{12}=\frac{-P_{2} \pm \sqrt{P_{2}^{2}-P_{1} P_{3}}}{P_{1}} \\
& Q_{1} \hat{\mu}_{13}^{2}+2 Q_{2} \hat{\mu}_{13}+Q_{3}=0 \\
& \hat{\mu}_{13}=\frac{-Q_{2} \pm \sqrt{Q_{2}^{2}-Q_{1} Q_{3}}}{Q_{1}} \\
& R_{1} \hat{\mu}_{14}^{2}+2 R_{2} \hat{\mu}_{14}+R_{3}=0 \\
& \hat{\mu}_{14}=\frac{-R_{2} \pm \sqrt{R_{2}^{2}-R_{1} R_{3}}}{R_{1}} \\
& S_{1} \hat{\mu}_{21}^{2}+2 S_{2} \hat{\mu}_{21}+S_{3}=0 \\
& \hat{\mu}_{21}=\frac{-S_{2} \pm \sqrt{S_{2}^{2}-S_{1} S_{3}}}{S_{1}} \\
& U_{1} \hat{\mu}_{22}^{2}+2 U_{2} \hat{\mu}_{22}+U_{3}=0 \\
& \hat{\mu}_{24}=\frac{-W_{2} \pm \sqrt{W_{2}^{2}-W_{1} W_{3}}}{W_{1}} \\
& \hat{\mu}_{23} \hat{\mu}_{24}^{2}+2 W_{2} \hat{\mu}_{24}+W_{3}=0 \\
& V_{1} \hat{\mu}_{23}^{2}+2 V_{2} \hat{\mu}_{23}+V_{3}=0 \\
& U_{1}-U_{1} U_{3} \\
& V_{2}^{2}-V_{1} V_{3} \\
& V_{1}
\end{aligned}
$$




\section{SINGH \& KHALID}

where

$$
\begin{aligned}
& T_{1}=A_{5} A_{8}+A_{2} A_{7}, \quad T_{2}=A_{3} A_{8}+A_{2} A_{6}, \quad T_{3}=A_{3} A_{7}-A_{5} A_{6}, \\
& P_{1}=A_{18} A_{14}-A_{17} A_{15}, \quad P_{2}=A_{3} A_{18}-A_{16} A_{15}, \quad P_{3}=A_{3} A_{17}-A_{16} A_{14}, \\
& Q_{1}=A_{28} A_{24}-A_{25} A_{27}, \quad Q_{2}=A_{3} A_{28}-A_{26} A_{25}, \quad Q_{3}=A_{3} A_{27}-A_{27} A_{24}, \\
& R_{1}=A_{39} A_{35}-A_{38} A_{36}, \quad R_{2}=A_{3} A_{38}-A_{37} A_{36}, \quad R_{3}=A_{3} A_{38}-A_{37} A_{35}, \\
& S_{1}=A_{45} A_{41}-A_{44} A_{42}, \quad S_{2}=A_{40} A_{45}-A_{43} A_{42}, \quad S_{3}=A_{40} A_{44}-A_{43} A_{41}, \\
& U_{1}=A_{53} A_{49}-A_{50} A_{52}, \quad U_{2}=A_{40} A_{53}-A_{51} A_{50}, \quad U_{3}=A_{40} A_{52}-A_{51} A_{49}, \\
& V_{1}=A_{61} A_{57}-A_{58} A_{60}, \quad V_{2}=A_{40} A_{61}-A_{58} A_{59}, \quad V_{3}=A_{40} A_{60}-A_{57} A_{59}, \\
& W_{1}=A_{69} A_{65}-A_{66} A_{68}, \quad W_{2}=A_{40} A_{69}-A_{66} A_{67}, \quad W_{3}=A_{40} A_{68}-A_{65} A_{67}
\end{aligned}
$$

The real values of $\hat{\mu}$ exit if and only if the quantity under the square root is greater than or equal to zero, i.e.

$$
\begin{aligned}
& \left(T_{2}^{2}-T_{1} T_{3}\right) \geq 0,\left(P_{2}^{2}-P_{1} P_{3}\right) \geq 0,\left(Q_{2}^{2}-Q_{1} Q_{3}\right) \geq 0,\left(R_{2}^{2}-R_{1} R_{3}\right) \geq 0, \\
& \left(S_{2}^{2}-S_{1} S_{3}\right) \geq 0,\left(U_{2}^{2}-U_{1} U_{3}\right) \geq 0,\left(V_{2}^{2}-V_{1} V_{3}\right) \geq 0,\left(W_{2}^{2}-W_{1} W_{3}\right) \geq 0
\end{aligned}
$$

For any situation which satisfies these conditions, two real values of $\hat{\mu}$ are possible. Hence, while choosing the value of $\hat{\mu}$, it is mentioned that $0 \leq \hat{\mu} \leq 1$ and all others values of $\hat{\mu}$ are inadmissible. In the case when both the values are admissible, the lowest one will be the best choice because it reduces the cost of the survey. Substituting the admissible value of $\hat{\mu}$, say $\mu^{(0)}$, from equations (57), (59), (61), (63), (65), (67), (69), and (71) into equations (41), (43), (45), (47), (49), (51), (53), and (55), respectively, gives the optimum value of the mean square error of the estimators $T_{i j}$ as

$$
\begin{gathered}
\mathrm{M}\left(T_{11}\right)_{\mathrm{opt}}^{*}=\frac{\left[A_{6}+\mu_{11}^{*} A_{7}+\mu_{11}^{* 2} A_{8}\right]}{\left[A_{3}+\mu_{11}^{*} A_{5}+\mu_{11}^{* 2} A_{2}\right]} \frac{S_{y}^{2}}{n} \\
\mathrm{M}\left(T_{12}\right)_{\mathrm{opt}}^{*}=\frac{\left[A_{16}+\mu_{12}^{*} A_{17}+\mu_{12}^{* 2} A_{18}\right]}{\left[A_{3}+\mu_{12}^{*} A_{14}+\mu_{12}^{* 2} A_{15}\right]} \frac{S_{y}^{2}}{n}
\end{gathered}
$$




$$
\begin{aligned}
& \mathrm{M}\left(T_{13}\right)_{\mathrm{opt}}^{*}=\frac{\left[A_{26}+\mu_{13}^{*} A_{27}+\mu_{13}^{* 2} A_{28}\right]}{\left[A_{3}+\mu_{13}^{*} A_{24}+\mu_{13}^{* 2} A_{25}\right]} \frac{S_{y}^{2}}{n} \\
& \mathrm{M}\left(T_{14}\right)_{\mathrm{opt}}^{*}=\frac{\left[A_{37}+\mu_{14}^{*} A_{38}+\mu_{14}^{* 2} A_{39}\right]}{\left[A_{3}+\mu_{14}^{*} A_{35}+\mu_{14}^{* 2} A_{36}\right]} \frac{S_{y}^{2}}{n} \\
& \mathrm{M}\left(T_{21}\right)_{\mathrm{opt}}^{*}=\frac{\left[A_{43}+\mu_{21}^{*} A_{44}+\mu_{21}^{* 2} A_{45}\right]}{\left[A_{40}+\mu_{21}^{*} A_{41}+\mu_{21}^{* 2} A_{42}\right]} \frac{S_{y}^{2}}{n} \\
& \mathrm{M}\left(T_{22}\right)_{\mathrm{opt}}^{*}=\frac{\left[A_{51}+\mu_{22}^{*} A_{52}+\mu_{22}^{* 2} A_{53}\right]}{\left[A_{40}+\mu_{22}^{*} A_{49}+\mu_{22}^{* 2} A_{50}\right]} \frac{S_{y}^{2}}{n} \\
& \mathrm{M}\left(T_{23}\right)_{\mathrm{opt}}^{*}=\frac{\left[A_{59}+\mu_{23}^{*} A_{60}+\mu_{23}^{* 2} A_{61}\right]}{\left[A_{40}+\mu_{23}^{*} A_{57}+\mu_{23}^{* 2} A_{58}\right]} \frac{S_{y}^{2}}{n} \\
& \mathrm{M}\left(T_{24}\right)_{\mathrm{opt}}^{*}=\frac{\left[A_{67}+\mu_{24}^{*} A_{68}+\mu_{24}^{* 2} A_{69}\right]}{\left[A_{40}+\mu_{24}^{*} A_{65}+\mu_{24}^{* 2} A_{66}\right]} \frac{S_{y}^{2}}{n}
\end{aligned}
$$

\section{Efficiency Comparisons}

The percent relative efficiencies of the estimators $T_{i j}(i=1,2 ; j=1,2,3,4)$ are calculated with respect to (i) sample mean estimator $\bar{y}_{n}$ when there is no matching and (ii) natural successive sampling estimator $\hat{\bar{Y}}=\phi^{*} \bar{y}_{u}+\left(1-\phi^{*}\right) \bar{y}_{m}^{\prime}$ when no auxiliary information is used on any occasion, where $\bar{y}_{m}^{\prime}=\bar{y}_{m}+\beta_{y x}\left(\bar{x}_{n}-\bar{x}_{m}\right)$. Because $\bar{y}_{n}$ and $\hat{\bar{Y}}$ are unbiased estimators of population $\bar{Y}$, therefore, following Sukhatme, Sukhatme, Sukhatme, and Ashok (1984), the variance of $\bar{y}_{n}$ and the optimum variance of $\hat{\bar{Y}}$ are given below:

$$
\mathrm{V}\left(\bar{y}_{n}\right)=\left(\frac{1}{n}-\frac{1}{N}\right) S_{y}^{2}
$$


and

$$
\mathrm{V}(\hat{\bar{Y}})_{\mathrm{opt}}=\left[1+\sqrt{1-\rho_{y x}^{2}}\right] \frac{S_{y}^{2}}{2 n}-\frac{S_{y}^{2}}{N}
$$

The optimum mean square errors of the estimators $T_{i j}$ are derived in equations (72)-(79), which involved six correlations. For the efficiency comparison of the suggested estimators, we introduce the assumption $\rho_{y z_{1}}=\rho_{y z_{2}}=\rho_{x z_{1}}=\rho_{x z_{2}}=\rho_{0}$, which is considered by Cochran (1977) and Feng and Zou (1997). Using the above assumptions, we have three correlations: $\rho_{y x}, \rho_{0}$, and $\rho_{z_{1} z_{2}}$.

The percent relative efficiencies $E_{i j}^{(1)}$ and $E_{i j}^{(2)}$ of the estimators $T_{i j}$ (under optimal conditions) with respect to $\bar{y}_{n}$ and $\hat{\bar{Y}}$ are defined below:

$$
E_{i j}^{(1)}=\frac{\mathrm{V}\left(\bar{y}_{n}\right)}{\mathrm{M}\left(T_{i j}\right)_{\mathrm{opt}}^{*}} \times 100 \quad \text { and } \quad E_{i j}^{(2)}=\frac{\mathrm{V}(\hat{\bar{Y}})_{\mathrm{opt}}}{\mathrm{M}\left(T_{i j}\right)_{\mathrm{opt}}^{*}} \times 100, \quad i=1,2 ; j=1,2,3,4
$$

\section{Empirical Study}

The expressions of the optimum values of $\mu_{i j}$, (i.e. $\left.\mu_{i j}^{(0)}\right)$ and the percent relative efficiencies $E_{i j}^{(1)}$ and $E_{i j}^{(2)}$ are in terms of population correlation coefficients $\rho_{y x}, \rho_{0}$, and $\rho_{z_{1} z_{2}}$. Tables 1-8 present the values of $\mu_{i j}^{(0)}, E_{i j}^{(1)}$, and $E_{i j}^{(2)}$ for a fixed value of $f=0.1$ and different choices of $\rho_{y x}, \rho_{0}$, and $\rho_{z_{1} z_{2}}$. 


\section{EFFICIENT CLASS OF ESTIMATORS IN TWO-OCCASION SAMPLING}

Table 1. Optimum values of $\mu_{11}^{(0)}$ and percent relative efficiencies of the estimator $T_{11}$ with respect to the estimators $\bar{y}_{n}$ and $\hat{\bar{Y}}$

\begin{tabular}{|c|c|c|c|c|c|c|}
\hline$\rho_{x y}$ & $\rho_{0}$ & 0.5 & 0.6 & 0.7 & 0.8 & 0.9 \\
\hline \multirow[t]{3}{*}{0.3} & $\mu_{11}^{(0)}$ & 0.4904 & 0.4776 & 0.4580 & 0.4259 & 0.3628 \\
\hline & $E_{11}^{(1)}$ & 130.4890 & 148.4939 & 177.9641 & 232.7923 & 370.6171 \\
\hline & $E_{11}^{(2)}$ & 127.1500 & 144.6940 & 173.4101 & 226.8353 & 361.1332 \\
\hline \multirow[t]{3}{*}{0.4} & $\mu_{11}^{(0)}$ & 0.4935 & 0.4772 & 0.4533 & 0.4156 & 0.3461 \\
\hline & $E_{11}^{(1)}$ & 131.4110 & 148.3820 & 175.9258 & 226.6202 & 352.3091 \\
\hline & $E_{11}^{(2)}$ & 125.3160 & 141.5000 & 167.7663 & 216.1094 & 335.9688 \\
\hline \multirow[t]{3}{*}{0.5} & $\mu_{11}^{(0)}$ & * & 0.4802 & 0.4519 & 0.4093 & 0.3348 \\
\hline & $E_{11}^{(1)}$ & ** & 149.3999 & 175.3590 & 222.8784 & 339.9487 \\
\hline & $E_{11}^{(2)}$ & ** & 138.2800 & 162.3069 & 206.2895 & 314.6462 \\
\hline \multirow[t]{3}{*}{0.6} & $\mu_{11}^{(0)}$ & 0.5104 & 0.4867 & 0.4539 & 0.4064 & 0.3274 \\
\hline & $E_{11}^{(1)}$ & 136.4283 & 151.6376 & 176.2118 & 221.1809 & 331.8836 \\
\hline & $E_{11}^{(2)}$ & 121.2696 & 134.7890 & 156.6327 & 196.6052 & 295.0077 \\
\hline \multirow[t]{3}{*}{0.7} & $\mu_{11}^{(0)}$ & 0.5258 & 0.4973 & 0.4594 & 0.4067 & 0.3231 \\
\hline & $E_{11}^{(1)}$ & 141.0230 & 155.3114 & 178.5626 & 221.3672 & 327.2309 \\
\hline & $E_{11}^{(2)}$ & 118.6272 & 130.6464 & 150.2051 & 186.2120 & 275.2635 \\
\hline \multirow[t]{3}{*}{0.8} & $\mu_{11}^{(0)}$ & 0.5481 & 0.5132 & 0.4690 & 0.4102 & 0.3215 \\
\hline & $E_{11}^{(1)}$ & 147.7268 & 160.8353 & 182.6430 & 223.4546 & 325.5421 \\
\hline & $E_{11}^{(2)}$ & 114.8986 & 125.0941 & 142.0557 & 173.7981 & 253.1994 \\
\hline \multirow[t]{3}{*}{0.9} & $\mu_{11}^{(0)}$ & 0.5810 & 0.5364 & 0.4835 & 0.4173 & 0.3226 \\
\hline & $E_{11}^{(1)}$ & 157.7795 & 168.9982 & 188.9145 & 227.6427 & 326.6647 \\
\hline & $E_{11}^{(2)}$ & 108.3323 & 116.0351 & 129.7097 & 156.3008 & 224.2897 \\
\hline
\end{tabular}

Note: * does not exist

${ }^{* *}$ no gain 


\section{SINGH \& KHALID}

Table 2. Optimum values of $\mu_{12}^{(0)}$ and percent relative efficiencies of the estimator $T_{12}$ with respect to the estimators $\bar{y}_{n}$ and $\hat{\bar{Y}}$

\begin{tabular}{|c|c|c|c|c|c|c|c|}
\hline$\rho_{x y}$ & $\rho_{0}$ & $\rho_{z_{1} z_{2}}$ & 0.4 & 0.5 & 0.6 & 0.7 & 0.8 \\
\hline \multirow[t]{15}{*}{0.3} & 0.4 & $\mu_{12}^{(0)}$ & 0.4925 & 0.4940 & 0.4955 & 0.4969 & 0.4984 \\
\hline & & $E_{12}^{(1)}$ & 123.7835 & 122.6390 & 121.5178 & 120.4192 & 119.3426 \\
\hline & & $E_{12}^{(2)}$ & 120.6160 & 119.5007 & 118.4082 & 117.3378 & 116.2887 \\
\hline & 0.5 & $\mu_{12}^{(0)}$ & 0.4786 & 0.4813 & 0.4840 & 0.4866 & 0.4891 \\
\hline & & $E_{12}^{(1)}$ & 140.3211 & 137.9947 & 135.7530 & 133.5914 & 131.5053 \\
\hline & & $E_{12}^{(2)}$ & 136.7304 & 134.4635 & 132.2792 & 130.1728 & 128.1402 \\
\hline & 0.6 & $\mu_{12}^{(0)}$ & 0.4571 & 0.4619 & 0.4666 & 0.4711 & 0.4754 \\
\hline & & $E_{12}^{(1)}$ & 168.3291 & 163.4167 & 158.8167 & 154.4987 & 150.4364 \\
\hline & & $E_{12}^{(2)}$ & 164.0217 & 159.2350 & 154.7527 & 150.5452 & 146.5869 \\
\hline & 0.7 & $\mu_{12}^{(0)}$ & 0.4211 & 0.4302 & 0.4388 & 0.4468 & 0.4544 \\
\hline & & $E_{12}^{(1)}$ & 222.7075 & 210.6427 & 199.9742 & 190.4620 & 181.9193 \\
\hline & & $E_{12}^{(2)}$ & 217.0085 & 205.2525 & 194.8570 & 185.5882 & 177.2641 \\
\hline & 0.8 & $\mu_{12}^{(0)}$ & 0.3485 & 0.3700 & 0.3885 & 0.4048 & 0.4192 \\
\hline & & $E_{12}^{(1)}$ & 373.1511 & 326.5884 & 291.6780 & 264.3665 & 242.3182 \\
\hline & & $E_{12}^{(2)}$ & 363.6024 & 318.2312 & 284.2141 & 257.6015 & 236.1174 \\
\hline \multirow[t]{12}{*}{0.4} & 0.4 & $\mu_{12}^{(0)}$ & 0.4970 & 0.4990 & 0.5010 & 0.5029 & 0.5049 \\
\hline & & $E_{12}^{(1)}$ & 126.3812 & 124.8064 & 123.2747 & 121.7842 & 120.3334 \\
\hline & & $E_{12}^{(2)}$ & 120.5196 & 119.0178 & 117.5572 & 116.1358 & 114.7522 \\
\hline & 0.5 & $\mu_{12}^{(0)}$ & 0.4794 & 0.4831 & 0.4867 & 0.4901 & 0.4935 \\
\hline & & $E_{12}^{(1)}$ & 143.3657 & 140.1532 & 137.0977 & 134.1874 & 131.4118 \\
\hline & & $E_{12}^{(2)}$ & 136.7164 & 133.6528 & 130.7390 & 127.9637 & 125.3168 \\
\hline & 0.6 & $\mu_{12}^{(0)}$ & 0.4525 & 0.4592 & 0.4655 & 0.4715 & 0.4772 \\
\hline & & $E_{12}^{(1)}$ & 172.5494 & 165.7021 & 159.4399 & 153.6874 & 148.3820 \\
\hline & & $E_{12}^{(2)}$ & 164.5465 & 158.0168 & 152.0450 & 146.5593 & 141.5000 \\
\hline & 0.7 & $\mu_{12}^{(0)}$ & 0.4083 & 0.4210 & 0.4327 & 0.4434 & 0.4533 \\
\hline & & $E_{12}^{(1)}$ & 231.1212 & 213.8394 & 199.2597 & 186.7676 & 175.9258 \\
\hline & & $E_{12}^{(2)}$ & 220.4017 & 203.9214 & 190.0179 & 178.1052 & 167.7663 \\
\hline
\end{tabular}




\section{EFFICIENT CLASS OF ESTIMATORS IN TWO-OCCASION SAMPLING}

Table 2 (continued).

\begin{tabular}{|c|c|c|c|c|c|c|c|}
\hline$\rho_{x y}$ & $\rho_{0}$ & $\rho_{z_{1} z_{2}}$ & 0.4 & 0.5 & 0.6 & 0.7 & 0.8 \\
\hline \multirow[t]{3}{*}{0.4} & 0.8 & $\mu_{12}^{(0)}$ & 0.3183 & 0.3500 & 0.3756 & 0.3971 & 0.4156 \\
\hline & & $E_{12}^{(1)}$ & 412.7503 & 337.6417 & 288.4129 & 253.2293 & 226.6202 \\
\hline & & $E_{12}^{(2)}$ & 393.6067 & 321.9818 & 275.0361 & 241.4844 & 216.1094 \\
\hline \multirow[t]{15}{*}{0.5} & 0.4 & $\mu_{12}^{(0)}$ & 0.5055 & 0.5081 & 0.5105 & 0.5130 & 0.5153 \\
\hline & & $E_{12}^{(1)}$ & 129.6446 & 127.6056 & 125.6365 & 123.7337 & 121.8937 \\
\hline & & $E_{12}^{(2)}$ & 119.9951 & 118.1079 & 116.2854 & 114.5242 & 112.8211 \\
\hline & 0.5 & $\mu_{12}^{(0)}$ & 0.4845 & 0.4891 & 0.4936 & 0.4979 & 0.5021 \\
\hline & & $E_{12}^{(1)}$ & 146.8017 & 142.6511 & 138.7540 & 135.0871 & 131.6295 \\
\hline & & $E_{12}^{(2)}$ & 135.8752 & 132.0335 & 128.4265 & 125.0325 & 121.8323 \\
\hline & 0.6 & $\mu_{12}^{(0)}$ & 0.4529 & 0.4613 & 0.4692 & 0.4766 & 0.4836 \\
\hline & & $E_{12}^{(1)}$ & 176.5595 & 167.7131 & 159.8085 & 152.6966 & 146.2588 \\
\hline & & $E_{12}^{(2)}$ & 163.4181 & 155.2301 & 147.9139 & 141.3314 & 135.3727 \\
\hline & 0.7 & $\mu_{12}^{(0)}$ & 0.4018 & 0.4182 & 0.4327 & 0.4459 & 0.4578 \\
\hline & & $E_{12}^{(1)}$ & 237.6206 & 215.0932 & 196.9329 & 181.9325 & 169.3007 \\
\hline & & $E_{12}^{(2)}$ & 219.9344 & 199.0838 & 182.2751 & 168.3912 & 156.6996 \\
\hline & 0.8 & $\mu_{12}^{(0)}$ & 0.2987 & 0.3405 & 0.3724 & 0.3981 & 0.4196 \\
\hline & & $E_{12}^{(1)}$ & 443.5963 & 338.7927 & 278.3913 & 238.3525 & 209.5417 \\
\hline & & $E_{12}^{(2)}$ & 410.5792 & 313.5763 & 257.6706 & 220.6119 & 193.9454 \\
\hline \multirow[t]{9}{*}{0.6} & 0.4 & $\mu_{12}^{(0)}$ & 0.5190 & 0.5220 & 0.5250 & 0.5279 & 0.5307 \\
\hline & & $E_{12}^{(1)}$ & 133.8029 & 131.2542 & 128.8104 & 126.4650 & 124.2119 \\
\hline & & $E_{12}^{(2)}$ & 118.9359 & 116.6704 & 114.4981 & 112.4133 & 110.4106 \\
\hline & 0.5 & $\mu_{12}^{(0)}$ & 0.4944 & * & 0.5053 & 0.5104 & 0.5153 \\
\hline & & $E_{12}^{(1)}$ & 150.7748 & ** & 140.8627 & 136.4283 & 132.2925 \\
\hline & & $E_{12}^{(2)}$ & 134.0221 & ** & 125.2113 & 121.2696 & 117.5934 \\
\hline & 0.6 & $\mu_{12}^{(0)}$ & 0.4585 & 0.4686 & 0.4780 & 0.4867 & 0.4948 \\
\hline & & $E_{12}^{(1)}$ & 180.3263 & 169.4813 & 160.0028 & 151.6376 & 144.1925 \\
\hline & & $E_{12}^{(2)}$ & 160.2900 & 150.6500 & 142.2247 & 134.7890 & 128.1711 \\
\hline
\end{tabular}

Note: * does not exist

${ }^{* *}$ no gain 


\section{SINGH \& KHALID}

Table 2 (continued).

\begin{tabular}{rrrrrrrr}
$\boldsymbol{\rho}_{x y}$ & $\boldsymbol{\rho}_{0}$ & $\boldsymbol{\rho}_{z_{1} z_{2}}$ & $\mathbf{0 . 4}$ & $\mathbf{0 . 5}$ & $\mathbf{0 . 6}$ & $\mathbf{0 . 7}$ & $\mathbf{0 . 8}$ \\
\hline 0.6 & 0.7 & $\mu_{12}^{(0)}$ & 0.4020 & 0.4217 & 0.4388 & 0.4539 & 0.4675 \\
& & $E_{12}^{(1)}$ & 241.4739 & 214.2100 & 193.1063 & 176.2118 & 162.3347 \\
& & $E_{12}^{(2)}$ & 214.6435 & 190.4089 & 171.6501 & 156.6327 & 144.2975 \\
& 0.8 & $\mu_{12}^{(0)}$ & 0.2912 & 0.3414 & 0.3778 & 0.4064 & 0.4299 \\
& & $E_{12}^{(1)}$ & 454.3984 & 328.5273 & 262.6792 & 221.1809 & 192.2438 \\
& & $E_{12}^{(2)}$ & 403.9097 & 292.0243 & 233.4927 & 196.6052 & 170.8834 \\
\hline
\end{tabular}

Table 3. Optimum values of $\mu_{13}^{(0)}$ and percent relative efficiencies of the estimator $T_{13}$ with respect to the estimators $\bar{y}_{n}$ and $\hat{\bar{Y}}$

\begin{tabular}{|c|c|c|c|c|c|c|c|}
\hline$\rho_{x y}$ & $\rho_{0}$ & $\rho_{z_{1} z_{2}}$ & 0.4 & 0.5 & 0.6 & 0.7 & 0.8 \\
\hline \multirow[t]{12}{*}{0.3} & 0.6 & $\mu_{13}^{(0)}$ & 0.7037 & 0.6649 & 0.6114 & 0.5327 & 0.4054 \\
\hline & & $E_{13}^{(1)}$ & 151.0761 & 150.5486 & 149.8111 & 148.7158 & 146.9328 \\
\hline & & $E_{13}^{(2)}$ & 147.2102 & 146.6961 & 145.9776 & 144.9103 & 143.1729 \\
\hline & 0.7 & $\mu_{13}^{(0)}$ & 0.6675 & 0.6328 & 0.5881 & 0.5282 & 0.4440 \\
\hline & & $E_{13}^{(1)}$ & 183.5163 & 182.4812 & 181.1345 & 179.3179 & 176.7449 \\
\hline & & $E_{13}^{(2)}$ & 178.8202 & 177.8117 & 176.4994 & 174.7293 & 172.2221 \\
\hline & 0.8 & $\mu_{13}^{(0)}$ & 0.6281 & 0.5954 & 0.5550 & 0.5035 & 0.4359 \\
\hline & & $E_{13}^{(1)}$ & 245.4888 & 243.2507 & 240.4555 & 236.8766 & 232.1453 \\
\hline & & $E_{13}^{(2)}$ & 239.2069 & 237.0260 & 234.3024 & 230.8151 & 226.2048 \\
\hline & 0.9 & $\mu_{13}^{(0)}$ & 0.5689 & 0.5362 & 0.4968 & 0.4483 & 0.3870 \\
\hline & & $E_{13}^{(1)}$ & 410.5928 & 403.8380 & 395.6042 & 385.3761 & 372.3671 \\
\hline & & $E_{13}^{(2)}$ & 400.0861 & 393.5041 & 385.4810 & 375.5145 & 362.8385 \\
\hline \multirow[t]{3}{*}{0.4} & 0.6 & $\mu_{13}^{(0)}$ & 0.6975 & 0.6444 & 0.5623 & 0.4187 & 0.1030 \\
\hline & & $E_{13}^{(1)}$ & 150.6510 & 149.8794 & 148.6716 & 146.5400 & 141.8383 \\
\hline & & $E_{13}^{(2)}$ & 143.6637 & 142.9279 & 141.7762 & 139.7434 & 135.2598 \\
\hline
\end{tabular}




\section{EFFICIENT CLASS OF ESTIMATORS IN TWO-OCCASION SAMPLING}

Table 3 (continued).

\begin{tabular}{|c|c|c|c|c|c|c|c|}
\hline$\rho_{x y}$ & $\rho_{0}$ & $\rho_{z_{1} z_{2}}$ & 0.4 & 0.5 & 0.6 & 0.7 & 0.8 \\
\hline \multirow[t]{9}{*}{0.4} & 0.7 & $\mu_{13}^{(0)}$ & 0.6559 & 0.6125 & 0.5535 & 0.4685 & 0.3359 \\
\hline & & $E_{13}^{(1)}$ & 181.4624 & 180.0003 & 177.9936 & 175.0855 & 170.5205 \\
\hline & & $E_{13}^{(2)}$ & 173.0461 & 171.6518 & 169.7381 & 166.9650 & 162.6117 \\
\hline & 0.8 & $\mu_{13}^{(0)}$ & 0.6120 & 0.5730 & 0.5232 & 0.4572 & 0.3659 \\
\hline & & $E_{13}^{(1)}$ & 239.7321 & 236.7151 & 232.8269 & 227.6469 & 220.4315 \\
\hline & & $E_{13}^{(2)}$ & 228.6132 & 225.7361 & 222.0283 & 217.0885 & 210.2078 \\
\hline & 0.9 & $\mu_{13}^{(0)}$ & 0.5474 & 0.5096 & 0.4630 & 0.4040 & 0.3271 \\
\hline & & $E_{13}^{(1)}$ & 393.0190 & 384.4791 & 373.8422 & 360.2781 & 342.4515 \\
\hline & & $E_{13}^{(2)}$ & 374.7906 & 366.6468 & 356.5033 & 343.5682 & 326.5684 \\
\hline \multirow[t]{12}{*}{0.5} & 0.6 & $\mu_{13}^{(0)}$ & 0.6890 & 0.6058 & 0.4431 & * & * \\
\hline & & $E_{13}^{(1)}$ & 150.7936 & 149.6352 & 147.3410 & 140.8400 & ** \\
\hline & & $E_{13}^{(2)}$ & 139.5700 & 138.4978 & 136.3743 & 130.3572 & $\star *$ \\
\hline & 0.7 & $\mu_{13}^{(0)}$ & 0.6427 & 0.5857 & 0.5016 & 0.3651 & 0.1052 \\
\hline & & $E_{13}^{(1)}$ & 180.1118 & 178.0750 & 175.0451 & 170.1042 & 160.6985 \\
\hline & & $E_{13}^{(2)}$ & 166.7060 & 164.8208 & 162.0165 & 157.4433 & 148.7377 \\
\hline & 0.8 & $\mu_{13}^{(0)}$ & 0.5958 & 0.5482 & 0.4849 & 0.3963 & 0.2636 \\
\hline & & $E_{13}^{(1)}$ & 235.1634 & 231.1981 & 225.8736 & 218.3839 & 207.1301 \\
\hline & & $E_{13}^{(2)}$ & 217.6601 & 213.9900 & 209.0617 & 202.1296 & 191.7134 \\
\hline & 0.9 & $\mu_{13}^{(0)}$ & 0.5274 & 0.4831 & 0.4272 & 0.3542 & 0.2548 \\
\hline & & $E_{13}^{(1)}$ & 378.6636 & 368.1038 & 354.6093 & 336.8406 & 312.4969 \\
\hline & & $E_{13}^{(2)}$ & 350.4796 & 340.7057 & 328.2156 & 311.7694 & 289.2377 \\
\hline \multirow[t]{6}{*}{0.6} & 0.6 & $\mu_{13}^{(0)}$ & 0.6703 & 0.4867 & * & * & * \\
\hline & & $E_{13}^{(1)}$ & 151.5113 & 149.3725 & 138.9485 & 172.8835 & 160.7811 \\
\hline & & $E_{13}^{(2)}$ & 134.6767 & 132.7756 & 123.5098 & 153.6742 & 142.9165 \\
\hline & 0.7 & $\mu_{13}^{(0)}$ & 0.6252 & 0.5438 & 0.4055 & 0.1192 & * \\
\hline & & $E_{13}^{(1)}$ & 179.3545 & 176.4170 & 171.4019 & 161.0463 & 127.7445 \\
\hline & & $E_{13}^{(2)}$ & 159.4263 & 156.8151 & 152.3572 & 143.1523 & 113.5507 \\
\hline
\end{tabular}

Note: * does not exist

** no gain 


\section{SINGH \& KHALID}

Table 3 (continued).

\begin{tabular}{rrrrrrrr}
$\boldsymbol{\rho}_{x y}$ & $\boldsymbol{\rho}_{0}$ & $\boldsymbol{\rho}_{z_{1} z_{2}}$ & $\mathbf{0 . 4}$ & $\mathbf{0 . 5}$ & $\mathbf{0 . 6}$ & $\mathbf{0 . 7}$ & $\mathbf{0 . 8}$ \\
\hline 0.6 & 0.8 & $\mu_{13}^{(0)}$ & 0.5778 & 0.5179 & 0.4333 & 0.3048 & 0.0863 \\
& & $E_{13}^{(1)}$ & 231.4250 & 226.1950 & 218.7627 & 207.4412 & 188.2306 \\
& & $E_{13}^{(2)}$ & 205.7111 & 201.0622 & 194.4557 & 184.3922 & 167.3161 \\
& 0.9 & $\mu_{13}^{(0)}$ & 0.5071 & 0.4546 & 0.3861 & 0.2927 & 0.1582 \\
& & $E_{13}^{(1)}$ & 366.2176 & 353.1933 & 336.0178 & 312.4628 & 278.3582 \\
& & $E_{13}^{(2)}$ & 325.5267 & 313.9496 & 298.6825 & 277.7447 & 247.4295 \\
\hline
\end{tabular}

Table 4. Optimum values of $\mu_{14}^{(0)}$ and percent relative efficiencies of the estimator $T_{14}$ with respect to the estimators $\bar{y}_{n}$ and $\hat{\bar{Y}}$

\begin{tabular}{|c|c|c|c|c|c|c|c|}
\hline$\rho_{x y}$ & $\rho_{0}$ & $\rho_{z_{1} z_{2}}$ & 0.4 & 0.5 & 0.6 & 0.7 & 0.8 \\
\hline \multirow[t]{9}{*}{0.3} & 0.6 & $\mu_{14}^{(0)}$ & 0.8051 & 0.7705 & 0.7147 & 0.6091 & 0.3344 \\
\hline & & $E_{14}^{(1)}$ & 158.3813 & 158.6421 & 159.0791 & 159.9277 & 162.1820 \\
\hline & & $E_{14}^{(2)}$ & 154.3284 & 154.5826 & 155.0083 & 155.8353 & 158.0319 \\
\hline & 0.7 & $\mu_{14}^{(0)}$ & 0.9949 & * & * & * & 0.6249 \\
\hline & & $E_{14}^{(1)}$ & 196.0800 & 196.0860 & 196.1594 & 196.7016 & 194.8757 \\
\hline & & $E_{14}^{(2)}$ & 191.0625 & 191.0683 & 191.1398 & 191.6681 & 189.8890 \\
\hline & 0.8 & $\mu_{14}^{(0)}$ & * & * & * & * & 0.4363 \\
\hline & & $E_{14}^{(1)}$ & 291.1143 & 308.1703 & ** & 241.4353 & 257.4570 \\
\hline & & $E_{14}^{(2)}$ & 283.6649 & 300.2845 & ** & 235.2572 & 250.8689 \\
\hline \multirow[t]{6}{*}{0.4} & 0.6 & $\mu_{14}^{(0)}$ & 0.7018 & 0.6591 & 0.5995 & 0.5101 & 0.3613 \\
\hline & & $E_{14}^{(1)}$ & 167.9310 & 169.3375 & 171.3226 & 174.3229 & 179.3608 \\
\hline & & $E_{14}^{(2)}$ & 160.1423 & 161.4835 & 163.3766 & 166.2377 & 171.0420 \\
\hline & 0.7 & $\mu_{14}^{(0)}$ & 0.7946 & 0.7546 & 0.6880 & 0.5545 & 0.1528 \\
\hline & & $E_{14}^{(1)}$ & 202.2086 & 203.0783 & 204.5727 & 207.6376 & 217.0745 \\
\hline & & $E_{14}^{(2)}$ & 192.8300 & 193.6595 & 195.0846 & 198.0073 & 207.0065 \\
\hline
\end{tabular}

Note: ${ }^{*}$ does not exist
${ }^{* \star}$ no gain 


\section{EFFICIENT CLASS OF ESTIMATORS IN TWO-OCCASION SAMPLING}

Table 4 (continued).

\begin{tabular}{|c|c|c|c|c|c|c|c|}
\hline$\rho_{x y}$ & $\rho_{0}$ & $\rho_{z_{1} z_{2}}$ & 0.4 & 0.5 & 0.6 & 0.7 & 0.8 \\
\hline \multirow[t]{3}{*}{0.4} & 0.8 & $\mu_{14}^{(0)}$ & 0.9823 & 0.9975 & * & * & 0.7975 \\
\hline & & $E_{14}^{(1)}$ & 277.8293 & 277.7785 & 277.8647 & 279.6778 & 276.2330 \\
\hline & & $E_{14}^{(2)}$ & 264.9434 & 264.8950 & 264.9772 & 266.7062 & 263.4212 \\
\hline \multirow[t]{9}{*}{0.5} & 0.6 & $\mu_{14}^{(0)}$ & 0.6889 & 0.6531 & 0.6067 & 0.5445 & 0.4562 \\
\hline & & $E_{14}^{(1)}$ & 192.4040 & 196.1477 & 201.0234 & 207.6273 & 217.0641 \\
\hline & & $E_{14}^{(2)}$ & 178.0833 & 181.5484 & 186.0612 & 192.1736 & 200.9080 \\
\hline & 0.7 & $\mu_{14}^{(0)}$ & 0.7286 & 0.6858 & 0.6240 & 0.5269 & 0.3524 \\
\hline & & $E_{14}^{(1)}$ & 225.7896 & 229.8347 & 235.7374 & 245.1169 & 262.2443 \\
\hline & & $E_{14}^{(2)}$ & 208.9840 & 212.7280 & 218.1914 & 226.8728 & 242.7254 \\
\hline & 0.8 & $\mu_{14}^{(0)}$ & 0.8061 & 0.7633 & 0.6870 & 0.5122 & * \\
\hline & & $E_{14}^{(1)}$ & 294.9041 & 297.7125 & 302.8927 & 315.1260 & ** \\
\hline & & $E_{14}^{(2)}$ & 272.9543 & 275.5537 & 280.3483 & 291.6711 & ** \\
\hline \multirow[t]{9}{*}{0.6} & 0.6 & $\mu_{14}^{(0)}$ & * & * & * & * & * \\
\hline & & $E_{14}^{(1)}$ & ** & ** & ** & $* *$ & ** \\
\hline & & $E_{14}^{(2)}$ & $* *$ & $* *$ & $* *$ & $* *$ & ** \\
\hline & 0.7 & $\mu_{14}^{(0)}$ & 0.8733 & 0.8568 & 0.8349 & 0.8043 & 0.7588 \\
\hline & & $E_{14}^{(1)}$ & 336.3958 & 354.1668 & 378.2031 & 412.4970 & 465.3477 \\
\hline & & $E_{14}^{(2)}$ & 299.0185 & 314.8150 & 336.1806 & 366.6640 & 413.6424 \\
\hline & 0.8 & $\mu_{14}^{(0)}$ & 0.8278 & 0.7963 & 0.7486 & 0.6676 & 0.5000 \\
\hline & & $E_{14}^{(1)}$ & 390.2813 & 409.1458 & 438.3804 & 489.5727 & 601.8214 \\
\hline & & $E_{14}^{(2)}$ & 346.9168 & 363.6851 & 389.6715 & 435.1758 & 534.9524 \\
\hline
\end{tabular}

Note: * does not exist

** no gain 


\section{SINGH \& KHALID}

Table 5. Optimum values of $\mu_{21}^{(0)}$ and percent relative efficiencies of the estimator $T_{21}$ with respect to the estimators $\bar{y}_{n}$ and $\hat{\bar{Y}}$

\begin{tabular}{|c|c|c|c|c|c|c|}
\hline$\rho_{y x}$ & $\rho_{0}$ & 0.5 & 0.6 & 0.7 & 0.8 & 0.9 \\
\hline \multirow[t]{3}{*}{0.3} & $\mu_{21}^{(0)}$ & * & 0.6759 & 0.5064 & 0.3831 & * \\
\hline & $E_{21}^{(1)}$ & 124.9983 & 160.8880 & 219.5400 & 360.7346 & 124.9983 \\
\hline & $E_{21}^{(2)}$ & 121.7997 & 156.7710 & 213.9222 & 351.5036 & 121.7997 \\
\hline \multirow[t]{3}{*}{0.4} & $\mu_{21}^{(0)}$ & * & 0.6497 & 0.4860 & 0.3639 & * \\
\hline & $E_{21}^{(1)}$ & 125.0000 & 159.3224 & 213.8407 & 342.9515 & 125.0000 \\
\hline & $E_{21}^{(2)}$ & 119.2024 & 151.9330 & 203.9227 & 327.0452 & 119.2024 \\
\hline \multirow[t]{3}{*}{0.5} & $\mu_{21}^{(0)}$ & * & 0.6431 & 0.4747 & 0.3511 & * \\
\hline & $E_{21}^{(1)}$ & 124.8775 & 158.8769 & 210.3709 & 330.9411 & 124.8775 \\
\hline & $E_{21}^{(2)}$ & 115.5828 & 147.0516 & 194.7129 & 306.3091 & 115.5828 \\
\hline \multirow[t]{3}{*}{0.6} & $\mu_{21}^{(0)}$ & * & 0.6531 & 0.4697 & 0.3428 & * \\
\hline & $E_{21}^{(1)}$ & 123.2817 & 159.5457 & 208.7938 & 323.1029 & 123.2817 \\
\hline & $E_{21}^{(2)}$ & 109.5837 & 141.8184 & 185.5945 & 287.2026 & 109.5837 \\
\hline \multirow[t]{3}{*}{0.7} & $\mu_{21}^{(0)}$ & * & 0.6845 & 0.4703 & 0.3381 & * \\
\hline & $E_{21}^{(1)}$ & 101.9803 & 161.3349 & 208.9670 & 318.5806 & 101.9803 \\
\hline & $E_{21}^{(2)}$ & * & 135.7134 & 175.7811 & 267.9869 & * \\
\hline \multirow[t]{3}{*}{0.8} & $\mu_{21}^{(0)}$ & * & 0.7589 & 0.4764 & 0.3364 & * \\
\hline & $E_{21}^{(1)}$ & ** & 164.1551 & 210.9059 & 316.9390 & ** \\
\hline & $E_{21}^{(2)}$ & ** & 127.6762 & 164.0379 & 246.5081 & ** \\
\hline \multirow[t]{3}{*}{0.9} & $\mu_{21}^{(0)}$ & 0.1445 & 1.0008 & 0.4892 & 0.3375 & 0.1445 \\
\hline & $E_{21}^{(1)}$ & 156.9299 & 166.6666 & 214.7873 & 318.0302 & 156.9299 \\
\hline & $E_{21}^{(2)}$ & 156.9299 & 114.4342 & 147.4741 & 218.3613 & 156.9299 \\
\hline
\end{tabular}

Note: * does not exist

no gain 


\section{EFFICIENT CLASS OF ESTIMATORS IN TWO-OCCASION SAMPLING}

Table 6. Optimum values of $\mu_{22}^{(0)}$ and percent relative efficiencies of the estimator $T_{22}$ with respect to the estimators $\bar{y}_{n}$ and $\hat{\bar{Y}}$

\begin{tabular}{|c|c|c|c|c|c|c|c|}
\hline$\rho_{y x}$ & $\rho_{0}$ & $\rho_{z_{1} z_{2}}$ & 0.4 & 0.5 & 0.6 & 0.7 & 0.8 \\
\hline \multirow[t]{9}{*}{0.3} & 0.6 & $\mu_{22}^{(0)}$ & 0.9900 & 0.9972 & * & * & * \\
\hline & & $E_{22}^{(1)}$ & 124.9957 & 124.9997 & 124.9987 & 124.9908 & 124.9724 \\
\hline & & $E_{22}^{(2)}$ & 121.7972 & 121.8010 & 121.8001 & 121.7923 & 121.7745 \\
\hline & 0.7 & $\mu_{22}^{(0)}$ & 0.7799 & 0.7656 & 0.7483 & 0.7267 & 0.6991 \\
\hline & & $E_{22}^{(1)}$ & 162.3520 & 162.2089 & 162.0254 & 161.7867 & 161.4698 \\
\hline & & $E_{22}^{(2)}$ & 158.1976 & 158.0581 & 157.8793 & 157.6467 & 157.3379 \\
\hline & 0.8 & $\mu_{22}^{(0)}$ & 0.6644 & 0.6406 & 0.6121 & 0.5771 & 0.5333 \\
\hline & & $E_{22}^{(1)}$ & 227.6079 & 226.4697 & 225.0821 & 223.3607 & 221.1785 \\
\hline & & $E_{22}^{(2)}$ & 221.7835 & 220.6745 & 219.3224 & 217.6451 & 215.5186 \\
\hline \multirow[t]{9}{*}{0.4} & 0.6 & $\mu_{22}^{(0)}$ & 0.9823 & 0.9903 & * & * & * \\
\hline & & $E_{22}^{(1)}$ & 124.9855 & 124.9962 & 125.0000 & 124.9931 & 124.9681 \\
\hline & & $E_{22}^{(2)}$ & 119.1886 & 119.1988 & 119.2024 & 119.1958 & 119.1720 \\
\hline & 0.7 & $\mu_{22}^{(0)}$ & 0.7652 & 0.7470 & 0.7240 & 0.6943 & 0.6541 \\
\hline & & $E_{22}^{(1)}$ & 161.2034 & 160.9714 & 160.6645 & 160.2494 & 159.6693 \\
\hline & & $E_{22}^{(2)}$ & 153.7267 & 153.5054 & 153.2128 & 152.8170 & 152.2638 \\
\hline & 0.8 & $\mu_{22}^{(0)}$ & 0.6488 & 0.6207 & 0.5861 & 0.5426 & 0.4860 \\
\hline & & $E_{22}^{(1)}$ & 223.0847 & 221.5307 & 219.5887 & 217.1067 & 213.8407 \\
\hline & & $E_{22}^{(2)}$ & 212.7380 & 211.2560 & 209.4041 & 207.0371 & 203.9227 \\
\hline \multirow[t]{6}{*}{0.5} & 0.6 & $\mu_{22}^{(0)}$ & * & * & * & * & * \\
\hline & & $E_{22}^{(1)}$ & 124.9920 & 124.9665 & 124.9117 & 124.8030 & 124.5768 \\
\hline & & $E_{22}^{(2)}$ & 115.6888 & 115.6652 & 115.6145 & 115.5139 & 115.3045 \\
\hline & 0.7 & $\mu_{22}^{(0)}$ & 0.7633 & 0.7412 & 0.7122 & 0.6720 & 0.6132 \\
\hline & & $E_{22}^{(1)}$ & 160.8262 & 160.5258 & 160.1070 & 159.5026 & 158.5823 \\
\hline & & $E_{22}^{(2)}$ & 148.8558 & 148.5778 & 148.1902 & 147.6308 & 146.7790 \\
\hline
\end{tabular}

Note: * does not exist

** no gain 


\section{SINGH \& KHALID}

Table 6 (continued).

\begin{tabular}{|c|c|c|c|c|c|c|c|}
\hline$\rho_{y x}$ & $\rho_{0}$ & $\rho_{z_{1} z_{2}}$ & 0.4 & 0.5 & 0.6 & 0.7 & 0.8 \\
\hline \multirow[t]{3}{*}{0.5} & 0.8 & $\mu_{22}^{(0)}$ & 0.6383 & 0.6052 & 0.5633 & 0.5084 & 0.4334 \\
\hline & & $E_{22}^{(1)}$ & 220.0004 & 218.0108 & 215.4460 & 212.0384 & 207.3241 \\
\hline & & $E_{22}^{(2)}$ & 203.6257 & 201.7842 & 199.4103 & 196.2563 & 191.8929 \\
\hline \multirow[t]{9}{*}{0.6} & 0.6 & $\mu_{22}^{(0)}$ & * & * & * & * & * \\
\hline & & $E_{22}^{(1)}$ & 124.6102 & 124.3316 & 123.8140 & 122.6460 & 118.2052 \\
\hline & & $E_{22}^{(2)}$ & 110.7646 & 110.5170 & 110.0569 & 109.0186 & 105.0713 \\
\hline & 0.7 & $\mu_{22}^{(0)}$ & 0.7720 & 0.7459 & 0.7091 & 0.6531 & 0.5579 \\
\hline & & $E_{22}^{(1)}$ & 161.2025 & 160.8682 & 160.3609 & 159.5457 & 158.0984 \\
\hline & & $\mu_{22}^{(0)}$ & 143.2911 & 142.9940 & 142.5430 & 141.8184 & 140.5319 \\
\hline & 0.8 & $E_{22}^{(1)}$ & 0.6312 & 0.5918 & 0.5399 & 0.4684 & 0.3633 \\
\hline & & $E_{22}^{(2)}$ & 218.1099 & 215.6326 & 212.3068 & 207.6494 & 200.7245 \\
\hline & & $E_{22}^{(2)}$ & 193.8754 & 191.6734 & 188.7172 & 184.5772 & 178.4218 \\
\hline
\end{tabular}

Note: ${ }^{*}$ does not exist

${ }^{* *}$ no gain

Table 7. Optimum values of $\mu_{23}^{(0)}$ and percent relative efficiencies of the estimator $T_{23}$ with respect to the estimators $\bar{y}_{n}$ and $\hat{\bar{Y}}$

\begin{tabular}{|c|c|c|c|c|c|c|c|}
\hline$\rho_{x y}$ & $\rho_{0}$ & $\rho_{z_{1} z_{2}}$ & 0.4 & 0.5 & 0.6 & 0.7 & 0.8 \\
\hline \multirow[t]{9}{*}{0.3} & 0.6 & $\mu_{23}^{(0)}$ & 0.9823 & 0.9922 & * & * & * \\
\hline & & $E_{23}^{(1)}$ & 124.9849 & 124.9975 & 124.9987 & 124.9800 & 124.9210 \\
\hline & & $E_{23}^{(2)}$ & 121.7866 & 121.7989 & 121.8001 & 121.7818 & 121.7244 \\
\hline & 0.7 & $\mu_{23}^{(0)}$ & 0.7909 & 0.7732 & 0.7500 & 0.7186 & 0.6734 \\
\hline & & $E_{23}^{(1)}$ & 162.4125 & 162.2399 & 161.9962 & 161.6432 & 161.1092 \\
\hline & & $E_{23}^{(2)}$ & 158.2565 & 158.0883 & 157.8509 & 157.5068 & 156.9865 \\
\hline & 0.8 & $\mu_{23}^{(0)}$ & 0.6770 & 0.6499 & 0.6161 & 0.5727 & 0.5151 \\
\hline & & $E_{23}^{(1)}$ & 228.0593 & 226.7618 & 225.1137 & 222.9660 & 220.0717 \\
\hline & & $E_{23}^{(2)}$ & 222.2234 & 220.9591 & 219.3532 & 217.2604 & 214.4402 \\
\hline
\end{tabular}

Note: * does not exist 


\section{EFFICIENT CLASS OF ESTIMATORS IN TWO-OCCASION SAMPLING}

Table 7 (continued).

\begin{tabular}{|c|c|c|c|c|c|c|c|}
\hline$\rho_{x y}$ & $\rho_{0}$ & $\rho_{z_{1} z_{2}}$ & 0.4 & 0.5 & 0.6 & 0.7 & 0.8 \\
\hline \multirow[t]{3}{*}{0.3} & 0.9 & $\mu_{23}^{(0)}$ & 0.5824 & 0.5510 & 0.5130 & 0.4661 & 0.4065 \\
\hline & & $E_{23}^{(1)}$ & 396.9078 & 391.1365 & 384.0539 & 375.1909 & 363.8247 \\
\hline & & $E_{23}^{(2)}$ & 386.7512 & 381.1276 & 374.2262 & 365.5901 & 354.5147 \\
\hline \multirow[t]{12}{*}{0.4} & 0.6 & $\mu_{23}^{(0)}$ & 0.9720 & 0.9832 & * & & * \\
\hline & & $E_{23}^{(1)}$ & 124.9612 & 124.9886 & 125.0000 & 124.9752 & 124.8351 \\
\hline & & $E_{23}^{(2)}$ & 119.1655 & 119.1915 & 119.2024 & 119.1788 & 119.0452 \\
\hline & 0.7 & $\mu_{23}^{(0)}$ & 0.7695 & 0.7447 & 0.7104 & 0.6598 & 0.5780 \\
\hline & & $E_{23}^{(1)}$ & 161.0824 & 160.7577 & 160.2779 & 159.5327 & 158.2762 \\
\hline & & $E_{23}^{(2)}$ & 153.6113 & 153.3017 & 152.8441 & 152.1335 & 150.9352 \\
\hline & 0.8 & $\mu_{23}^{(0)}$ & 0.6562 & 0.6228 & 0.5797 & 0.5221 & 0.4409 \\
\hline & & $E_{23}^{(1)}$ & 223.1409 & 221.2761 & 218.8258 & 215.4921 & 210.7338 \\
\hline & & $E_{23}^{(2)}$ & 212.7915 & 211.0132 & 208.6765 & 205.4975 & 200.9598 \\
\hline & 0.9 & $\mu_{23}^{(0)}$ & 0.5598 & 0.5232 & 0.4779 & 0.4203 & 0.3448 \\
\hline & & $E_{23}^{(1)}$ & 380.3266 & 372.9314 & 363.6460 & 351.6988 & 335.8323 \\
\hline & & $E_{23}^{(2)}$ & 362.6869 & 355.6346 & 346.7799 & 335.3868 & 320.2562 \\
\hline \multirow[t]{12}{*}{0.5} & 0.6 & $\mu_{23}^{(0)}$ & 0.9976 & * & * & * & * \\
\hline & & $E_{23}^{(1)}$ & 124.9997 & 124.9813 & 124.8680 & 124.3137 & ** \\
\hline & & $E_{23}^{(2)}$ & 115.6960 & 115.6789 & 115.5741 & 115.0610 & ** \\
\hline & 0.7 & $\mu_{23}^{(0)}$ & 0.7562 & 0.7220 & 0.6703 & 0.5831 & 0.4045 \\
\hline & & $E_{23}^{(1)}$ & 160.3641 & 159.8555 & 159.0351 & 157.5812 & 154.4997 \\
\hline & & $E_{23}^{(2)}$ & 148.4281 & 147.9574 & 147.1980 & 145.8524 & 143.0002 \\
\hline & 0.8 & $\mu_{23}^{(0)}$ & 0.6382 & 0.5967 & 0.5407 & 0.4612 & 0.3394 \\
\hline & & $E_{23}^{(1)}$ & 219.3604 & 216.8137 & 213.3149 & 208.2627 & 200.4141 \\
\hline & & $E_{23}^{(2)}$ & 203.0333 & 200.6762 & 197.4378 & 192.7616 & 185.4972 \\
\hline & 0.9 & $\mu_{23}^{(0)}$ & 0.5392 & 0.4962 & 0.4416 & 0.3698 & 0.2712 \\
\hline & & $E_{23}^{(1)}$ & 366.8796 & 357.6462 & 345.7340 & 329.8765 & 307.8596 \\
\hline & & $E_{23}^{(2)}$ & 339.5726 & 331.0264 & 320.0009 & 305.3236 & 284.9455 \\
\hline
\end{tabular}

Note: * does not exist

${ }^{* *}$ no gain 


\section{SINGH \& KHALID}

Table 7 (continued).

\begin{tabular}{rrrrrrrr}
$\boldsymbol{\rho}_{x y}$ & $\boldsymbol{\rho}_{0}$ & $\boldsymbol{\rho}_{z_{1} z_{2}}$ & $\mathbf{0 . 4}$ & $\mathbf{0 . 5}$ & $\mathbf{0 . 6}$ & $\mathbf{0 . 7}$ & $\mathbf{0 . 8}$ \\
\hline 0.6 & 0.6 & $\mu_{23}^{(0)}$ & $*$ & $*$ & $*$ & 0.3855 & 0.7019 \\
& & $E_{23}^{(1)}$ & 124.7031 & 124.0416 & 118.1805 & 128.9701 & 127.2655 \\
& $E_{23}^{(2)}$ & 110.8472 & 110.2592 & 105.0493 & 114.6401 & 113.1249 \\
& 0.7 & $\mu_{23}^{(0)}$ & 0.7472 & 0.6979 & 0.6105 & 0.4136 & $*$ \\
& & $E_{23}^{(1)}$ & 160.2552 & 159.4993 & 158.0692 & 154.7028 & 139.7695 \\
& & $E_{23}^{(2)}$ & 142.4491 & 141.7772 & 140.5060 & 137.5136 & 124.2396 \\
& & $\mu_{23}^{(0)}$ & 0.6204 & 0.5673 & 0.4913 & 0.3731 & 0.1641 \\
& 0.8 & $E_{23}^{(1)}$ & 216.4316 & 212.9830 & 207.9427 & 199.9911 & 185.7935 \\
& $E_{23}^{(2)}$ & 192.3837 & 189.3182 & 184.8379 & 177.7699 & 165.1498 \\
& $\mu_{23}^{(0)}$ & 0.5187 & 0.4675 & 0.4002 & 0.3078 & 0.1730 \\
& & $E_{23}^{(1)}$ & 355.3191 & 343.8360 & 328.5210 & 307.2312 & 275.8615 \\
& $E_{23}^{(2)}$ & 315.8392 & 305.6320 & 292.0187 & 273.0944 & 245.2102 \\
\hline
\end{tabular}

Note: * does not exist

Table 8. Optimum values of $\mu_{24}^{(0)}$ and percent relative efficiencies of the estimator $T_{24}$ with respect to the estimators $\bar{y}_{n}$ and $\hat{\bar{Y}}$

\begin{tabular}{rrrrrrrrr}
$\boldsymbol{\rho}_{x y}$ & $\boldsymbol{\rho}_{0}$ & $\boldsymbol{\rho}_{z_{1} z_{2}}$ & $\mathbf{0 . 4}$ & $\mathbf{0 . 5}$ & $\mathbf{0 . 6}$ & $\mathbf{0 . 7}$ & $\mathbf{0 . 8}$ \\
\hline 0.3 & 0.6 & $\mu_{24}^{(0)}$ & 0.4532 & 0.3098 & 0.0440 & $*$ & $*$ \\
& & $E_{24}^{(1)}$ & 136.6962 & 139.2998 & 144.1264 & $*$ & $*$ \\
& & $E_{24}^{(2)}$ & 133.1982 & 135.7352 & 140.4383 & $*$ & $*$ \\
& 0.7 & $\mu_{24}^{(0)}$ & 0.7551 & 0.6649 & 0.3941 & $*$ & $*$ \\
& & $E_{24}^{(1)}$ & 169.3926 & 170.0616 & 172.1917 & $*$ & 163.2345 \\
& & $E_{24}^{(2)}$ & 165.0580 & 165.7099 & 167.7855 & $*$ & 159.0575 \\
& 0.8 & $\mu_{24}^{(0)}$ & $*$ & $*$ & $*$ & 0.3754 & 0.6200 \\
& & $E_{24}^{(1)}$ & 256.1296 & 268.0813 & 195.2127 & 235.5873 & 240.5105 \\
& $E_{24}^{(2)}$ & 249.5754 & 261.2213 & 190.2173 & 229.5587 & 234.3560 \\
\hline
\end{tabular}

Note: * does not exist 


\section{EFFICIENT CLASS OF ESTIMATORS IN TWO-OCCASION SAMPLING}

Table 8 (continued).

\begin{tabular}{|c|c|c|c|c|c|c|c|}
\hline$\rho_{x y}$ & $\rho_{0}$ & $\rho_{z_{1} z_{2}}$ & 0.4 & 0.5 & 0.6 & 0.7 & 0.8 \\
\hline \multirow[t]{9}{*}{0.4} & 0.6 & $\mu_{24}^{(0)}$ & 0.5321 & 0.4530 & 0.3359 & 0.1446 & * \\
\hline & & $E_{24}^{(1)}$ & 146.2100 & 149.2274 & 153.7028 & 160.9946 & ** \\
\hline & & $E_{24}^{(2)}$ & 139.4287 & 142.3061 & 146.5740 & 153.5276 & ** \\
\hline & 0.7 & $\mu_{24}^{(0)}$ & 0.6792 & 0.6045 & 0.4705 & 0.1598 & * \\
\hline & & $E_{24}^{(1)}$ & 178.5578 & 180.7188 & 184.6569 & 193.8518 & ** \\
\hline & & $E_{24}^{(2)}$ & 170.2762 & 172.3370 & 176.0924 & 184.8608 & ** \\
\hline & 0.8 & $\mu_{24}^{(0)}$ & 0.9197 & 0.9098 & 0.8804 & * & * \\
\hline & & $E_{24}^{(1)}$ & 250.9050 & 250.7622 & 250.6741 & ** & 250.0000 \\
\hline & & $E_{24}^{(2)}$ & 239.2679 & 239.1317 & 239.0477 & ** & 238.4049 \\
\hline \multirow[t]{9}{*}{0.5} & 0.6 & $\mu_{24}^{(0)}$ & 0.6089 & 0.5596 & 0.4940 & 0.4022 & 0.2648 \\
\hline & & $E_{24}^{(1)}$ & 168.3577 & 173.0219 & 179.2419 & 187.9328 & 200.9038 \\
\hline & & $E_{24}^{(2)}$ & 155.8267 & 160.1439 & 165.9008 & 173.9449 & 185.9504 \\
\hline & 0.7 & $\mu_{24}^{(0)}$ & 0.6731 & 0.6175 & 0.5345 & 0.3974 & 0.1273 \\
\hline & & $E_{24}^{(1)}$ & 201.7462 & 206.7289 & 214.2127 & 226.6348 & 251.1222 \\
\hline & & $E_{24}^{(2)}$ & 186.7302 & 191.3420 & 198.2688 & 209.7663 & 232.4311 \\
\hline & 0.8 & $\mu_{24}^{(0)}$ & 0.7744 & 0.7212 & 0.6220 & 0.3724 & * \\
\hline & & $E_{24}^{(1)}$ & 270.1604 & 273.6626 & 280.3900 & 297.7184 & ** \\
\hline & & $E_{24}^{(2)}$ & 250.0522 & 253.2938 & 259.5205 & 275.5591 & ** \\
\hline \multirow[t]{9}{*}{0.6} & 0.6 & $\mu_{24}^{(0)}$ & * & * & * & * & * \\
\hline & & $E_{24}^{(1)}$ & ** & ** & ** & ** & ** \\
\hline & & $E_{24}^{(2)}$ & ** & ** & ** & ** & ** \\
\hline & 0.7 & $\mu_{24}^{(0)}$ & 0.8636 & 0.8452 & 0.8205 & 0.7855 & 0.7317 \\
\hline & & $E_{24}^{(1)}$ & 300.9492 & 317.4336 & 339.8368 & 371.9968 & 421.9851 \\
\hline & & $E_{24}^{(2)}$ & 267.5104 & 282.1632 & 302.0772 & 330.6638 & 375.0979 \\
\hline & 0.8 & $\mu_{24}^{(0)}$ & 0.8186 & 0.7845 & 0.7321 & 0.6406 & 0.4406 \\
\hline & & $E_{24}^{(1)}$ & 359.7332 & 377.8316 & 406.1680 & 456.5742 & 570.5409 \\
\hline & & $E_{24}^{(2)}$ & 319.7629 & 335.8503 & 361.0382 & 405.8437 & 507.1475 \\
\hline
\end{tabular}

Note: * does not exist

${ }^{* *}$ no gain 


\section{Interpretations of Empirical Results}

The following interpretations can be drawn from Tables 1-8:

\section{Table 1}

(a) For fixed values of $\rho_{x y}$, the values of $E_{11}^{(1)}$ and $E_{11}^{(2)}$ are increasing, whereas for increasing values of $\rho_{0}, \mu_{11}^{(0)}$ decreases. These trends are highly considerable in the estimation procedure of the population mean because it not only reduces the cost of the sample survey but also make the estimator more precise.

(b) For fixed values of $\rho_{0}$, the values of $\mu_{11}^{(0)}$ and $E_{11}^{(1)}$ slightly decrease at the very starting level and then significantly increase with increasing values of $\rho_{x y}$, while a decreasing pattern is observed in $E_{11}^{(2)}$.

(c) The lowest value of $\mu_{11}^{(0)}$ is 0.3215 . This implies that only $30 \%$ of the sample on the current occasion has to be replaced by fresh units, which leads to a considerable depletion in the cost of the sample survey.

\section{Table 2}

(a) For fixed values of $\rho_{y x}$ and $\rho_{0}$ the values of $\mu_{12}^{(0)}, E_{12}^{(1)}$, and $E_{12}^{(2)}$ are all observed in a decreasing pattern with very slow rate, with the increasing values of $\rho_{z_{1} z_{2}}$.

(b) For fixed values of $\rho_{y x}$ and $\rho_{z_{1} z_{2}}$ the values of $\mu_{12}^{(0)}$ are found to be in a decreasing pattern while $E_{12}^{(1)}$ and $E_{12}^{(2)}$ are increasing with increasing values of $\rho_{0}$. These trends are also highly considerable in the estimation procedure of the population mean because it not only reduces the cost of the sample survey but also make the estimator more precise.

(c) For fixed values of $\rho_{0}$ and $\rho_{z_{1} z_{2}}$ the values of $E_{12}^{(2)}$ are decreasing while the values of $\mu_{12}^{(0)}$ and $E_{12}^{(1)}$ do not show any regular pattern with increasing values of $\rho_{y x}$.

(e) The lowest value of $\mu_{12}^{(0)}$ is 0.1358 . This implies that only $13 \%$ of the sample on the current occasion has to be replaced by fresh units, which leads to a considerable depletion in the cost of the sample survey. 


\section{EFFICIENT CLASS OF ESTIMATORS IN TWO-OCCASION SAMPLING}

\section{Table 3}

(a) For fixed values of $\rho_{y x}$ and $\rho_{0}$ the values $\mu_{13}^{(0)}, E_{13}^{(1)}$, and $E_{13}^{(2)}$ are all in a decreasing pattern, observed with increasing values of $\rho_{z_{1} z_{2}}$.

(b) For fixed values of $\rho_{y x}$ and $\rho_{z_{1} z_{2}}$ the values of $\mu_{13}^{(0)}$ are found to follow a decreasing pattern while $E_{13}^{(1)}$ and $E_{13}^{(2)}$ are increasing with the increasing value of $\rho_{0}$. This pattern indicates that the smaller fresh sample on the current occasion is required if information on the highly correlated auxiliary variables is available, these behaviors are highly correlated.

(c) For the fixed values of $\rho_{0}$ and $\rho_{z_{1} z_{2}}$ the values of $\mu_{13}^{(0)}$ and $E_{13}^{(1)}$ are decreasing with increasing values of $\rho_{y x}$ while the values of $E_{13}^{(2)}$ are increasing with increasing values of $\rho_{x y}$.

(d) The lowest value of $\mu_{13}^{(0)}$ is 0.0863 . This implies that only $8 \%$ of the sample on the current occasion has to be replaced by fresh units, which leads to a considerable depletion in the cost of the sample survey.

\section{Table 4}

a) For fixed values of $\rho_{y x}$ and $\rho_{0}$ the optimum values of $\mu_{14}^{(0)}$ follow a decreasing pattern with the increasing values of $\rho_{z_{1} z_{2}}$, while the values of $E_{14}^{(1)}$ and $E_{14}^{(2)}$ are increasing with increasing values of $\rho_{z_{1} z_{2}}$. These trends are also highly considerable in the estimation procedure of the population mean because it not only reduces the cost of the sample survey but also makes the estimator more precise.

(b) For fixed values of $\rho_{y x}$ and $\rho_{z_{1} z_{2}}$ the values $\mu_{14}^{(0)}, E_{14}^{(1)}$, and $E_{14}^{(2)}$ are all in an increasing pattern observed with the increasing values of $\rho_{0}$.

(c) For fixed values of $\rho_{0}$ and $\rho_{z_{1} z_{2}}$ the optimum values of $\mu_{14}^{(0)}$ are all decreasing at the very starting level and then a decremental pattern is observed with the increasing values of $\rho_{y x}$. The values of $E_{14}^{(1)}$ and $E_{14}^{(2)}$ are increasing at the very starting level and then a decremental pattern is observed with the increasing values of $\rho_{y x}$.

(d) The minimum value of $\mu_{14}^{(0)}$ is 0.1528 . This implies that only $15 \%$ of the sample on the current occasion has to be replaced by fresh units, which leads to an appreciable reduction in the cost of the sample survey. 


\section{SINGH \& KHALID}

\section{Table 5}

(a) For fixed values of $\rho_{y x}$, the values of $E_{21}^{(1)}$ and $E_{21}^{(2)}$ are increasing, whereas for increasing values of $\rho_{0}, \mu_{21}^{(0)}$ decreases. This trend is also highly desirable in the efficient estimation procedure of the population mean because it not only reduces the cost of the sample survey, but also makes the estimator more precise.

(b) For fixed values of $\rho_{0}$, the optimum values of $\mu_{21}^{(0)}$ and $E_{21}^{(1)}$ are found in decreasing patterns at the very starting level and then an incremental pattern is observed, while the values of $E_{21}^{(2)}$ are decreasing with the increasing values of $\rho_{y x}$. (c) The minimum value of $\mu_{21}^{(0)}$ is 0.3305 . This implies that only $33 \%$ of the sample on the current occasion has to be replaced by fresh units, which leads to a considerable depletion in the cost of the sample survey.

\section{Table 6}

(a) For fixed values of $\rho_{y x}$ and $\rho_{0}$ the values of $\mu_{22}^{(0)}, E_{22}^{(1)}$, and $E_{22}^{(2)}$ have a decreasing patterns observed with the increase in values of $\rho_{z_{1} z_{2}}$.

(b) For fixed values of $\rho_{y x}$ and $\rho_{z_{1} z_{2}}$ the values $\mu_{22}^{(0)}$ are found in a decreasing pattern, and $E_{22}^{(1)}$ and $E_{22}^{(2)}$ are increasing with the increasing values of $\rho_{0}$. These patterns also indicate the smaller fresh sample at current occasion is required, if the information on highly correlated auxiliary variables is available, these behaviors are highly correlated.

(c) For fixed values of $\rho_{0}$ and $\rho_{z_{1} z_{2}}$ the values of $\mu_{22}^{(0)}, E_{22}^{(1)}$, and $E_{22}^{(2)}$ decreased with an increase in values of $\rho_{y x}$.

(d) The minimum value of $\mu_{22}^{(0)}$ is 0.4334 . This shows that only $43 \%$ of the sample on the current occasion has to be replaced by fresh units, which leads to a considerable depletion in the cost of the sample survey.

\section{Table 7}

(a) For fixed values of $\rho_{y x}$ and $\rho_{0}$ the values $\mu_{23}^{(0)}, E_{23}^{(1)}$, and $E_{23}^{(2)}$ are all decreasing with increasing values of $\rho_{z_{1} z_{2}}$.

(b) For fixed values of $\rho_{y x}$ and $\rho_{z_{1} z_{2}}$ the values $\mu_{23}^{(0)}$ are found in a decreasing pattern while $E_{23}^{(1)}$ and $E_{23}^{(2)}$ are increasing with the increasing values of $\rho_{0}$. These 


\section{EFFICIENT CLASS OF ESTIMATORS IN TWO-OCCASION SAMPLING}

sequences also indicate the smaller fresh sample on the current occasion is required, if the information on highly correlated auxiliary variables is available, these behaviors are highly correlated.

(d) For fixed values of $\rho_{0}$ and $\rho_{z_{1} z_{2}}$ the values of $\mu_{23}^{(0)}, E_{23}^{(1)}$, and $E_{23}^{(2)}$ are all decreasing with the increasing values of $\rho_{y x}$.

(e) The lowest value of $\mu_{23}^{(0)}$ is 0.2712 . This shows that only $27 \%$ of the sample on the current occasion is substituted with fresh units, which leads to a considerable depletion in the cost of the sample survey.

\section{Table 8}

(a) For fixed values of $\rho_{y x}$ and $\rho_{0}$ the optimum values of $\mu_{24}^{(0)}$ are found to be in an approximately decreasing pattern while $E_{24}^{(1)}$ and $E_{24}^{(2)}$ are increased with increasing values of $\rho_{z_{1} z_{2}}$. These results indicate the smaller fresh sample on the current occasion is required if the information on highly correlated auxiliary variables is available, these behaviors are highly correlated.

(b) For fixed values of $\rho_{0}$ and $\rho_{z_{1} z_{2}}$ the values of $\mu_{24}^{(0)}$ are found to be in an increasing pattern while $E_{24}^{(1)}$ and $E_{24}^{(2)}$ decreases with increasing values of $\rho_{y x}$. This pattern also indicates the smaller fresh sample at current occasion is required if the information on highly correlated auxiliary variables is available, these behaviors are highly correlated.

(c) For fixed values of $\rho_{y x}$ and $\rho_{z_{1} z_{2}}$ the values of $\mu_{24}^{(0)}, E_{24}^{(1)}$, and $E_{24}^{(2)}$ increased with the increase in $\rho_{y x}$.

(d) The lowest value of $\mu_{24}^{(0)}$ is 0.1273 . This shows that only $12 \%$ of the sample on the current occasion has to be replaced by fresh units, which leads to a considerable depletion in the cost of the sample survey.

\section{Mutual Comparisons of the Proposed Estimators}

Mutual comparisons of the proposed estimators $T_{i j}(i=1,2 ; j=1,2,3,4)$ for their optimum mean square errors have been made with respect to the natural successive sampling estimator $\hat{\bar{Y}}$ (when there is no auxiliary information used on any occasion). For this comparison, we fix $\rho_{z_{1} z_{2}}$ and $f$ for different choices of $\rho_{y x}$ and $\rho_{0}$. The percent relative efficiencies $E_{i j}^{(2)}$ are given in Tables 9, 10, and 11 . 


\section{SINGH \& KHALID}

Table 9. The percent relative efficiencies of the proposed estimators $T_{i j}$ with respect to $\hat{\bar{Y}}$ for $\rho_{z_{1} z_{2}}=0.4$

\begin{tabular}{|c|c|c|c|c|}
\hline$\rho_{x y}$ & $\rho_{0}$ & 0.6 & 0.7 & 0.8 \\
\hline \multirow[t]{8}{*}{0.3} & $E_{11}^{(2)}$ & 144.6940 & 173.4101 & 226.8353 \\
\hline & $E_{12}^{(2)}$ & 147.1126 & 178.4900 & 238.5757 \\
\hline & $E_{13}^{(2)}$ & 147.2100 & 178.8200 & 239.2069 \\
\hline & $E_{14}^{(2)}$ & 154.3280 & 191.0625 & 283.6649 \\
\hline & $E_{21}^{(2)}$ & 121.7979 & 156.7710 & 213.9272 \\
\hline & $E_{22}^{(2)}$ & 121.7972 & 158.0581 & 157.8791 \\
\hline & $E_{23}^{(2)}$ & 121.7866 & 158.2565 & 222.7264 \\
\hline & $E_{24}^{(2)}$ & 131.1982 & 165.0580 & 249.5754 \\
\hline \multirow[t]{8}{*}{0.4} & $E_{11}^{(2)}$ & 141.5000 & 167.7663 & 216.1094 \\
\hline & $E_{12}^{(2)}$ & 144.0046 & 173.2253 & 228.5805 \\
\hline & $E_{13}^{(2)}$ & 143.6637 & 173.0461 & 228.6123 \\
\hline & $E_{14}^{(2)}$ & 160.1423 & 192.8300 & 264.9443 \\
\hline & $E_{21}^{(2)}$ & 119.2024 & 151.9330 & 203.9227 \\
\hline & $E_{22}^{(2)}$ & 119.1886 & 153.7261 & 212.7951 \\
\hline & $E_{23}^{(2)}$ & 119.1656 & 153.6113 & 212.7951 \\
\hline & $E_{24}^{(2)}$ & 139.4278 & 170.2762 & 239.2628 \\
\hline \multirow[t]{8}{*}{0.5} & $E_{11}^{(2)}$ & 138.2800 & 162.3069 & 206.2895 \\
\hline & $E_{12}^{(2)}$ & 140.5170 & 167.6554 & 218.5372 \\
\hline & $E_{13}^{(2)}$ & 139.5700 & 166.7060 & 217.6601 \\
\hline & $E_{14}^{(2)}$ & 178.0833 & 208.9840 & 272.9543 \\
\hline & $E_{21}^{(2)}$ & 115.5828 & 147.0516 & 194.7129 \\
\hline & $E_{22}^{(2)}$ & 115.6888 & 148.5858 & 203.6252 \\
\hline & $E_{23}^{(2)}$ & 115.6960 & 148.4281 & 203.0330 \\
\hline & $E_{24}^{(2)}$ & 155.8267 & 186.7302 & 250.0522 \\
\hline \multirow[t]{2}{*}{0.6} & $E_{11}^{(2)}$ & 134.7890 & 156.6327 & 196.6052 \\
\hline & $E_{12}^{(2)}$ & 136.3244 & 161.4230 & 207.8138 \\
\hline
\end{tabular}




\section{EFFICIENT CLASS OF ESTIMATORS IN TWO-OCCASION SAMPLING}

Table 9 (continued).

\begin{tabular}{rrrrr}
$\boldsymbol{\rho}_{x y}$ & $\boldsymbol{\rho}_{0}$ & $\mathbf{0 . 6}$ & $\mathbf{0 . 7}$ & $\mathbf{0 . 8}$ \\
\hline 0.6 & $E_{13}^{(2)}$ & 134.6767 & 159.4263 & 205.7110 \\
& $E_{14}^{(2)}$ & $* *$ & 229.0180 & 346.9100 \\
& $E_{21}^{(2)}$ & 109.5831 & 141.8148 & 185.5940 \\
& $E_{22}^{(2)}$ & 110.7646 & 143.2929 & 193.8170 \\
& $E_{23}^{(2)}$ & 110.8472 & 142.4991 & 192.3600 \\
& $E_{24}^{(2)}$ & $* *$ & 267.5109 & 319.7620 \\
\hline
\end{tabular}

Note: ** no gain

Table 10. The percent relative efficiencies of the proposed estimators $T_{i j}$ with respect to $\hat{\bar{Y}}$ for $\rho_{z_{1} z_{2}}=0.6$

\begin{tabular}{rrrrr}
$\rho_{x y}$ & $\rho_{0}$ & 0.6 & 0.7 & 0.8 \\
\hline 0.3 & $E_{11}^{(2)}$ & 144.6940 & 173.4101 & 226.8353 \\
& $E_{12}^{(2)}$ & 150.1449 & 176.7197 & 234.3901 \\
& $E_{13}^{(2)}$ & 145.9776 & 176.4994 & 234.3024 \\
& $E_{14}^{(2)}$ & 155.0083 & 191.1398 & $* *$ \\
& $E_{21}^{(2)}$ & 121.7979 & 156.7710 & 213.9272 \\
& $E_{22}^{(2)}$ & 121.8001 & 157.8791 & 219.3224 \\
& $E_{23}^{(2)}$ & 121.8001 & 157.8509 & 219.3534 \\
& $E_{24}^{(2)}$ & 140.4383 & 167.7855 & 190.2173 \\
0.4 & $E_{11}^{(2)}$ & 141.5000 & 167.7663 & 216.1094 \\
& $E_{12}^{(2)}$ & 143.0230 & 170.9484 & 223.2368 \\
& $E_{13}^{(2)}$ & 141.7762 & 169.7381 & 222.0283 \\
& $E_{14}^{(2)}$ & 163.3766 & 195.0846 & 264.9772 \\
& $E_{21}^{(2)}$ & 119.2024 & 151.9330 & 203.9227 \\
& $E_{22}^{(2)}$ & 119.2024 & 153.2120 & 209.4014 \\
& $E_{23}^{(2)}$ & 119.2024 & 152.8441 & 208.6765 \\
& $E_{24}^{(2)}$ & 146.5740 & 176.0924 & 239.0477 \\
\hline
\end{tabular}

Note: ${ }^{* *}$ no gain 


\section{SINGH \& KHALID}

Table 10 (continued).

\begin{tabular}{rrrrr}
$\rho_{x y}$ & $\boldsymbol{\rho}_{0}$ & $\mathbf{0 . 6}$ & $\mathbf{0 . 7}$ & $\mathbf{0 . 8}$ \\
\hline 0.5 & $E_{11}^{(2)}$ & 138.2800 & 162.3069 & 206.2895 \\
& $E_{12}^{(2)}$ & 139.4486 & 164.8864 & 211.9974 \\
& $E_{13}^{(2)}$ & 136.3743 & 162.0156 & 209.0617 \\
& $E_{14}^{(2)}$ & 186.0612 & 218.1914 & 280.3483 \\
& $E_{21}^{(2)}$ & 115.5828 & 147.0516 & 194.7129 \\
& $E_{22}^{(2)}$ & 115.6652 & 148.1902 & 199.4103 \\
& $E_{23}^{(2)}$ & 115.5741 & 147.1980 & 197.4378 \\
& $E_{24}^{(2)}$ & 165.9008 & 198.2688 & 259.5205 \\
0.6 & $E_{11}^{(2)}$ & 134.7890 & 156.6327 & 196.6052 \\
& $E_{12}^{(2)}$ & 135.9615 & 160.0569 & 204.4225 \\
& $E_{13}^{(2)}$ & 123.5098 & 152.3572 & 194.4557 \\
& $E_{14}^{(2)}$ & $* *$ & 336.1806 & 389.6715 \\
& $E_{21}^{(2)}$ & 109.5831 & 141.8148 & 185.5940 \\
& $E_{22}^{(2)}$ & 110.0569 & 142.5430 & 188.7172 \\
& $E_{23}^{(2)}$ & 105.0493 & 140.5060 & 184.8370 \\
& $E_{24}^{(2)}$ & $* *$ & 302.0722 & 361.0382 \\
\hline & & & &
\end{tabular}

Note: ${ }^{* \star}$ no gain

Table 11. The percent relative efficiencies of the proposed estimators $T_{i j}$ with respect to $\hat{\bar{Y}}$ for $\rho_{z_{1} z_{2}}=0.8$

\begin{tabular}{rrrrr}
$\boldsymbol{\rho}_{x y}$ & $\boldsymbol{\rho}_{0}$ & $\mathbf{0 . 6}$ & $\mathbf{0 . 7}$ & $\mathbf{0 . 8}$ \\
\hline 0.3 & $E_{11}^{(2)}$ & 144.6940 & 173.4101 & 226.8353 \\
& $E_{12}^{(2)}$ & 144.9749 & 173.9691 & 228.0913 \\
& $E_{13}^{(2)}$ & 143.1729 & 172.2221 & 226.2048 \\
& $E_{14}^{(2)}$ & 158.0319 & 189.0319 & 250.8689 \\
& $E_{21}^{(2)}$ & 121.7979 & 156.7710 & 213.9222 \\
& $E_{22}^{(2)}$ & 121.7745 & 157.3379 & 215.5186 \\
& $E_{23}^{(2)}$ & 121.7244 & 156.9865 & 214.4402 \\
\hline
\end{tabular}




\section{EFFICIENT CLASS OF ESTIMATORS IN TWO-OCCASION SAMPLING}

Table 11 (continued).

\begin{tabular}{rlrrr}
$\boldsymbol{\rho}_{x y}$ & $\boldsymbol{\rho}_{0}$ & $\mathbf{0 . 6}$ & $\mathbf{0 . 7}$ & $\mathbf{0 . 8}$ \\
\hline 0.3 & $E_{24}^{(2)}$ & $* *$ & 159.0575 & 234.3560 \\
0.4 & $E_{11}^{(2)}$ & 141.5000 & 167.7663 & 216.1094 \\
& $E_{12}^{(2)}$ & 141.1649 & 167.1260 & 214.7253 \\
& $E_{13}^{(2)}$ & 135.2598 & 162.6117 & 210.2078 \\
& $E_{14}^{(2)}$ & 171.0420 & 207.0065 & 263.4212 \\
& $E_{21}^{(2)}$ & 119.2024 & 151.9330 & 203.9227 \\
& $E_{22}^{(2)}$ & 119.1720 & 152.2683 & 203.9227 \\
& $E_{23}^{(2)}$ & 119.0452 & 150.9352 & 200.9598 \\
& $E_{24}^{(2)}$ & $* *$ & $* *$ & 238.4095 \\
& $E_{11}^{(2)}$ & 138.2800 & 162.3069 & 206.2895 \\
& $E_{12}^{(2)}$ & 136.7974 & 159.6454 & 200.7785 \\
& $E_{13}^{(2)}$ & $* *$ & 148.7377 & 191.7134 \\
& $E_{14}^{(2)}$ & 200.9080 & 242.7254 & $* *$ \\
& $E_{21}^{(2)}$ & 115.5828 & 147.0516 & 194.7129 \\
& $E_{22}^{(2)}$ & 115.3045 & 146.7790 & 191.8929 \\
& $E_{23}^{(2)}$ & $* *$ & 143.0020 & 185.4972 \\
& $E_{24}^{(2)}$ & 232.4431 & $* *$ & $* *$ \\
& $E_{11}^{(2)}$ & 134.7890 & 156.6327 & 196.6052 \\
& $E_{12}^{(2)}$ & 130.1055 & 150.6192 & 185.0459 \\
& $E_{13}^{(2)}$ & 142.9165 & 113.5507 & 167.3161 \\
& $E_{14}^{(2)}$ & $* *$ & 413.6424 & 534.9524 \\
& $E_{21}^{(2)}$ & 109.5831 & 141.8148 & 185.5940 \\
& $E_{22}^{(2)}$ & 105.0713 & 140.5319 & 178.4218 \\
& $E_{23}^{(2)}$ & 113.1249 & 124.2396 & 165.1498 \\
& $E_{24}^{(2)}$ & $* *$ & 375.0979 & 507.1475 \\
\hline & & & & \\
& & &
\end{tabular}

Note: ** no gain 


\section{SINGH \& KHALID}

\section{Conclusion}

The use of modified ratio and regression type estimators for the estimation of the current population mean on the current occasion in two-occasion successive sampling is justified through empirical results. The use of highly correlated auxiliary variable $z_{1}$ and $z_{2}$ which are firmly fixed over time is greatly rewarding; it not only reduces the cost of the survey but also enhances the precision of the estimates as well. Also, it can be seen in the section of mutual comparisons that the proposed estimators $T_{14}$ and $T_{24}$ are more efficient than the other proposed estimators. The suggested class of estimators, especially $T_{14}$ and $T_{24}$, are more rewarding in terms of precision and also reduce the cost of the survey. The proposed class of estimators may be recommended to survey practitioners for their real-life practical problems.

\section{Acknowledgements}

The Authors are thankful to the reviewers for their encouraging and positive comments, which helped us in preparing the improved version of the paper. The Authors are also thankful to the IIT(ISM), Dhanbad, for providing the financial assistance and necessary infrastructure to carry out the present research work.

\section{References}

Biradar, R. S., \& Singh, H. P. (2001). Successive sampling using auxiliary information on both the occasions. Calcutta Statistical Associations Bulletin, 51(3-4), 243-251. doi: 10.1177/0008068320010307

Chaturvedi, D. K., \& Tripathi, T. P. (1983). Estimation of population ratio on two occasions using multivariate auxiliary information. Journal of Indian Statistical Association, 21, 113-120.

Cochran, W. G. (1977). Sampling techniques (3rd ed.). New Delhi, India: Wiley Eastern Limited.

Das, A. K. (1982). Estimation of population ratio on two occasions. Journal of the Indian Society of Agricultural Statistics, 34(2), 1-9.

Eckler, A. R. (1955). Rotation sampling. The Annals of Mathematical Statistics, 26(4), 664-685. doi: 10.1214/aoms/1177728427 


\section{EFFICIENT CLASS OF ESTIMATORS IN TWO-OCCASION SAMPLING}

Feng, S., \& Zou, G. (1997). Sample rotation method with auxiliary variable. Communication in Statistics - Theory and Methods, 26(6), 1497-1509. doi: 10.1080/03610929708831996

Gupta, P. C. (1979). Sampling on two successive occasions. Journal of Statistical Research, 13(13), 7-16.

Jessen, R. J. (1942). Statistical investigation of a sample surveys for obtaining farm facts. Iowa Agriculture and Home Economics Research Station Research Bulletin, 26(304), 1-104. Retrieved from https://lib.dr.iastate.edu/researchbulletin/vol26/iss304/1/

Patterson, H. D. (1950). Sampling on successive occasions with partial replacement of units. Journal of the Royal Statistical Society. Series B (Methodological), 12(2), 241-255. Available from https://www.jstor.org/stable/2983982

Reddy, V. N. (1978). A study on the use of prior knowledge on certain population parameters in estimation. Sankhya, Series $C$, 40, 29-37.

Sen, A. R. (1971). Successive sampling with two auxiliary variables. Sankhyā: The Indian Journal of Statistics, Series B, 33(3/4), 371-378. Available from https://www.jstor.org/stable/25051764

Sen, A. R. (1972). Successive sampling with $p(p \geq 1)$ auxiliary variables. The Annals of Mathematical Statistics, 43(6), 2301-2304. doi:

10.1214/aoms/1177690878

Sen, A. R. (1973). Note: Theory and application of sampling on repeated occasions with several auxiliary variables. Biometrics 29(2), 381-385. doi: $10.2307 / 2529401$

Singh, G. N. (2003). Estimation of population mean using auxiliary information on recent occasions in h-occasion successive sampling. Statistics in Transition, 6(4), 523-532. Retrieved from https://pts.stat.gov.pl/en/journals/statistics-in-transition/

Singh, G. N. (2005). On the use of chain-type ratio estimator in successive sampling. Statistics in Transition, 7(1), 21-26. Retrieved from https://pts.stat.gov.pl/en/journals/statistics-in-transition/

Singh, G. N., Homa, F. S., \& Murya, S. (2013). Exponential method of estimation in two-occasion successive sampling. International Journal of Statistics \& Economics, 12(3), 26-39. Available from http://ceser.in/ceserp/index.php/bse/article/view/2164 


\section{SINGH \& KHALID}

Singh, G. N., \& Karna, J. P. (2009). Estimation of population mean on the current in two-occasion successive sampling. METRON - International Journal of Statistics, 67(1), 87-103.

Singh, G. N., \& Prasad, S. (2010). Some estimators of population mean in two-occasion rotation patterns. Association for the Advancement of Modeling \& Simulation Techniques in Enterprises, 12(1), 25-44.

Singh, G. N., Prasad, S., \& Karna, J. P. (2011). Some classes of estimators for population mean at current occasion in two-occasion successive sampling. Journal of Statistical Research, 45(1) 21-36.

Singh, G. N., \& Priyanka, K. (2008). Search of good rotation patterns to improve the precision of estimates at current occasions. Communication in Statistics - Theory and Methods, 37(3), 337-348. doi: 10.1080/03610920701653052

Singh, G. N., \& Singh, V. K. (2001). On the use of auxiliary information in successive sampling. Journal of the Indian Society of Agricultural Statistics, 54(1), 1-12.

Singh, H. P., Kim, J. M., \& Tarray, T. A. (2016). A family of estimators of population variance in two-occasion rotation patterns. Communications in Statistics - Theory and Methods, 45(14), 4106-4116. doi:

10.1080/03610926.2014.915047

Singh, H. P., Srivastava, M. K., Srivastava, N., Tarray, T. A., Singh, V., \& Dixit, S. (2015). Chain regression-type estimator using multiple auxiliary information in successive sampling. Hacettepe Journal of Mathematics and Statistics, 44(5), 1247-1256. doi: 10.15672/HJMS.2014287479

Singh, H. P., Vishwakarma, G. K. (2009). A general procedure for estimating population mean in successive sampling. Communication in Statistics - Theory and Methods, 38(2), 293-308. doi: 10.1080/03610920802169594

Singh, V. K., Singh, G. N., \& Shukla, D. (1991). An efficient family of ratio-cum-difference type estimators in successive sampling over two occasions. Journal of Statistical Research, 41, 149-159.

Sukhatme, P. V., Sukhatme, B. V., Sukhatme, S., \& Ashok, C. (1984). Sampling theory of surveys with applications. Ames, IA: Iowa State University Press. 